\title{
Effect of an angular trajectory kick in a high-gain free-electron laser
}

\author{
Panagiotis Baxevanis, Zhirong Huang, and Gennady Stupakov \\ SLAC National Accelerator Laboratory, Menlo Park, California 94025, USA
}

(Received 6 December 2016; published 18 April 2017)

\begin{abstract}
In a free-electron laser, a transverse momentum offset (or "kick") results in an oscillation of the centroid of the electron beam about the undulator axis. Studying the influence of this effect on the free-electron laser (FEL) interaction is important both from a tolerance point of view and for its potential diagnostic applications. In this paper, we present a self-consistent theoretical analysis of a high-gain FEL driven by such a "kicked" beam. In particular, we derive a solution to the three-dimensional, linearized initial value problem of the FEL through an orthogonal expansion technique and also describe a variational method for calculating the average FEL growth rate. Our results are benchmarked with GENESIS simulations and provide a robust theoretical background for a comparison with previous analytical results.
\end{abstract}

DOI: 10.1103/PhysRevAccelBeams.20.040703

\section{INTRODUCTION}

In a typical configuration for a high-gain, $\mathrm{x}$-ray freeelectron laser (XFEL) [1-4], the orbit of the driving electron beam is actively controlled through focusing and feedback elements in order to avoid large centroid excursions from the undulator axis, which can significantly degrade the performance of the machine. There are, however, several cases where off-axis radiation propagation can occur, mainly through the introduction (deliberate or accidental) of angular trajectory errors (or "kicks"). To begin with, random errors in the focusing system can cause such kicks, an issue of particular importance for high reprate machines [such as Linac Coherent Light Source II (LCLS-II)] due to the danger that the mis-steered photon beam poses to collimators and similar x-ray beam line elements [5]. Moreover, wake fields and coherent synchrotron radiation can induce a correlation of the transverse slope of the electron beam with the internal bunch position, leading to a "tilt" in phase space [6]. It can be shown that the study of this effect requires a thorough understanding of the basic physics of an FEL driven by a "kicked" beam. The examples mentioned so far emphasize the tolerance aspect of the issue under consideration. On the other hand, an angular kick is often an essential part of many FEL-related diagnostic or experimental procedures. The standard gain length measurement procedure in LCLS-I employs such kicks in order to terminate the interaction at a given location and record the corresponding radiation pulse energy. Other applications include schemes such as the DELTA variable polarization undulator [7] (where different polarization

Published by the American Physical Society under the terms of the Creative Commons Attribution 4.0 International license. Further distribution of this work must maintain attribution to the author(s) and the published article's title, journal citation, and DOI. components are separated by means of an angular kick to the bunched $e$-beam) or proposed fast gain length measurement techniques using energy-loss data from kicked beams [8].

In existing literature, the standard analytical result regarding the influence of a single angular trajectory error $\theta_{0}$ is the so-called Tanaka gain length formula [9]. Specifically, it states that the kick increases the FEL power gain length $L_{G}$ from an initial value of $L_{G 0}$ to

$$
L_{G}=\frac{L_{G 0}}{1-\left(\theta_{0} / \theta_{c}\right)^{2}}
$$

where $\theta_{c}=\sqrt{\lambda_{r} / L_{G 0}}$ is the critical angle and $\lambda_{r}$ the radiation wavelength (the idea being that any kick larger than $\theta_{c}$ completely suppresses the FEL effect). Alternatively, one may interpret this relation as a quadratic decrease in the FEL growth rate, with a definite cutoff at $\theta_{0}=\theta_{c}$. Though this result appears reliable enough for many purposes, its derivation has been based on rather simple arguments and assumptions more appropriate for a fully bunched beam (a model more likely to be realized in the saturation regime). Thus, the question arises whether the formula is equally applicable in other stages of the interaction (such as the linear regime) and whether the simple expression for the critical angle is accurate enough in such cases. Moreover, we deem it highly desirable to study the problem at hand using the same rigorous techniques that are used in the theoretical analysis of more standard FEL configurations $[10,11]$.

We also point out that some recent studies [12] have suggested that Ref. [9] is conceptually wrong and that no coherent radiation intensity degradation should occur after a single kick of a microbunched beam. Although we disagree with these studies and would also like to point out the recent LCLS beam diverting experiment using the DELTA undulator [7] (which actually showed radiation 
intensity degradation), the motivation for this paper did not originate from this debate. Instead, we want to address the growth rate degradation due to an angular kick that affects the trajectory in the entire undulator. The linear analysis presented in this paper allows us to obtain a more accurate formula in order to compare with Eq. (1).

In this paper, we seek to develop the framework for such a systematic consideration of the impact that a single angular kick has on the operation of a high-gain FEL. To this end, we use the Maxwell-Vlasov equations of the FEL (including a transverse momentum offset) to derive a selfconsistent, fully 3D equation satisfied by the radiation amplitude in the linear regime of the interaction. We then employ an orthogonal expansion method in order to derive a semianalytical, approximate solution to the linearized, initial value problem (IVP) of the FEL. The basis elements are suitably constructed to allow for off-axis radiation propagation and yield accurate results even with a few expansion modes. Moreover, we develop a periodic analysis technique which enables us to calculate the average FEL growth rate (over many betatron oscillations) by means of a variational procedure similar to that which is used in a standard eigenvalue problem. After a comparison with simulation, we use the results obtained from the IVP in order to extract a value for the average FEL growth rate, which is then compared with the outcome of the periodic analysis. This procedure reveals an encouraging consistency between all the components used and allows us to critically assess the applicability of Tanaka's formula in various scenarios.

\section{THEORY}

\section{A. FEL equations}

We begin our treatment with a review of the equations of motion for a single electron in the combined field of the undulator and the FEL radiation. The latter is assumed to be linearly polarized in the $x$ direction and its electric field is given by $E_{r}=(1 / 2) E_{\nu}(\mathbf{x}, z) e^{i \nu k_{r}(z-c t)}+$ c.c. Here, c.c. stands for complex conjugate, $E_{\nu}(\mathbf{x}, z)$ is the radiation amplitude [a function of the transverse position $\mathbf{x}=(x, y)$ and the longitudinal position $z$ along the undulator], and $\nu$ is the frequency of the radiation scaled by the resonant frequency $\omega_{r}\left(\nu=\omega / \omega_{r}\right)$. The latter quantity is given by $\omega_{r}=c k_{r}=2 \pi c / \lambda_{r}$, where $k_{r}\left(\lambda_{r}\right)$ is the resonant wave number (wavelength), in turn defined through the FEL resonance condition $\lambda_{r}=\lambda_{u}\left(1+K_{0}^{2} / 2\right) /\left(2 \gamma_{0}^{2}\right)$. Here, $\lambda_{u}=$ $2 \pi / k_{u}$ is the undulator period, $K_{0}=q_{e} B_{0} /\left(m_{e} c k_{u}\right)$ is the dimensionless undulator parameter $\left(q_{e} / m_{e}\right.$ is the electron charge/mass, $B_{0}$ is the peak, on-axis magnetic field), and $\gamma_{0}$ is the average value for the electron Lorentz factor. The radiation amplitude is assumed to be a slowly varying function of $z$, i.e., it changes little over an undulator period $\left[\left|\left(\lambda_{u} / E_{\nu}\right) \partial E_{\nu} / \partial z\right| \ll 1\right]$. In the context of our model, after averaging out the fast wiggle effect, the transverse motion of the electrons is governed by the relations $x^{\prime \prime}=-k_{\beta x}^{2} x$ and $y^{\prime \prime}=-k_{\beta y}^{2} y$ (where a prime denotes differentiation with respect to $z$ ). The focusing strengths $k_{\beta x}$ and $k_{\beta y}$ are, in general, assumed to be unequal but constant. For the case of an undulator with parabolically shaped pole faces and no external focusing, this representation is exact. In fact, the natural focusing strengths satisfy $k_{\beta x}^{2}+k_{\beta y}^{2}=k_{n}^{2}=K_{0}^{2} k_{u}^{2}$ / $\left(2 \gamma_{0}^{2}\right)$. If external focusing is to be added, the model can still be valid provided the focusing lattice is treated in the smooth approximation [10]. The transverse equations of motion can also be written as

$$
\begin{array}{ll}
\frac{d x}{d z}=p_{x}, & \frac{d p_{x}}{d z}=-k_{\beta x}^{2} x, \\
\frac{d y}{d z}=p_{y}, & \frac{d p_{y}}{d z}=-k_{\beta y}^{2} y,
\end{array}
$$

a form which also introduces the transverse slopes $p_{x}, p_{y}$. The longitudinal dynamics of the system is determined by the generalized pendulum equations, namely

$\frac{d \theta}{d z}=\theta^{\prime}=2 k_{u} \delta-\frac{k_{r}}{2}\left(p_{x}^{2}+p_{y}^{2}+k_{\beta x}^{2} x^{2}+k_{\beta y}^{2} y^{2}\right)$,

$\frac{d \delta}{d z}=\kappa_{1} E_{\nu}(\mathbf{x}, z) e^{-i \Delta \nu k_{u} z} e^{i \nu \theta}+$ c.c.

Here, $\theta=k_{u} z+k_{r}(z-c t)+Q_{0} \sin \left(2 k_{u} z\right)$ is the phase variable $\left[Q_{0}=K_{0}^{2} /\left(4+2 K_{0}^{2}\right)\right], \delta=\gamma / \gamma_{0}-1$ is the energy deviation, $\Delta \nu=\nu-1$ is the frequency detuning, and $\kappa_{1}=q_{e} K_{0}[J J] /\left(4 \gamma_{0}^{2} m_{e} c^{2}\right)$, with $[J J]=J_{0}\left(Q_{0}\right)-J_{1}\left(Q_{0}\right)$ being a well-known factor arising from the wiggle averaging. The quadratic term on the right-hand side of Eq. (3) represents the influence of the transverse motion on the longitudinal phase space dynamics.

The self-consistent interaction between the electron beam and the generated FEL radiation in the field of the undulator can be studied using the Maxwell-Vlasov equations for the combined system [10]. The 6D phase space state of the electron beam is characterized by a distribution function of the form $f=f\left(\theta, \delta, x, y, p_{x}, p_{y}, z\right)$, whose value is constant along individual phase space trajectories ( $f$ is akin to a phase space density). Following the standard approach, we confine our attention to the linear (or exponential gain) regime of the interaction, in which $f$ can be expressed as $f=f_{0}+f_{1}$, where $f_{0}$ is a background distribution and $f_{1}$ is a small perturbation due to the microbunching. In view of the fact that frequencies are decoupled in the linear regime, $f_{1}$ is written as $f_{1}=$ $\hat{f}_{\nu} e^{i \nu \theta}+$ c.c., where $\hat{f}_{\nu}=\hat{f}_{\nu}\left(\delta, x, y, p_{x}, p_{y}, z\right)$ is another amplitude quantity (analogous to $E_{\nu}$ ). It should be stressed that, in the context of this perturbation method, both $E_{\nu}$ and $\hat{f}_{\nu}$ are assumed to be first-order (small) quantities, apart from slowly varying functions of $z$. Up to the onset of 
saturation, the operation of the FEL can be adequately described via the following linearized, frequency-domain, Maxwell-Vlasov equations [13]:

$$
\begin{gathered}
\left(\frac{\partial}{\partial z}+\frac{1}{2 i k_{r}}\left[\frac{\partial^{2}}{\partial x^{2}}+\frac{\partial^{2}}{\partial y^{2}}\right]\right) E_{\nu}(x, y, z) \\
=-\kappa_{2} e^{i \Delta \nu k_{u} z} \int d p_{x} d p_{y} d \delta \hat{f}_{\nu}\left(\delta, x, y, p_{x}, p_{y}, z\right), \\
\frac{\partial \hat{f}_{\nu}}{\partial z}+p_{x} \frac{\partial \hat{f}_{\nu}}{\partial x}+p_{y} \frac{\partial \hat{f}_{\nu}}{\partial y}-k_{\beta x}^{2} x \frac{\partial \hat{f}_{\nu}}{\partial p_{x}}-k_{\beta y}^{2} y \frac{\partial \hat{f}_{\nu}}{\partial p_{y}}+i \theta^{\prime} \hat{f}_{\nu} \\
=-\kappa_{1} E_{\nu} e^{-i \Delta \nu k_{u} z} \frac{\partial f_{0}}{\partial \delta},
\end{gathered}
$$

where $\kappa_{2}=q_{e} K_{0}[J J] /\left(2 \varepsilon_{0} \gamma_{0}\right)$ and we recall that $\theta^{\prime}$ is given by Eq. (3). The unperturbed distribution $f_{0}$ satisfies the zeroth-order Vlasov equation

$\frac{\partial f_{0}}{\partial z}+p_{x} \frac{\partial f_{0}}{\partial x}+p_{y} \frac{\partial f_{0}}{\partial y}-k_{\beta x}^{2} x \frac{\partial f_{0}}{\partial p_{x}}-k_{\beta y}^{2} y \frac{\partial f_{0}}{\partial p_{y}}=0$,

while the same quantity is normalized according to the relation

$$
\int d p_{x} d p_{y} d \delta \int d x d y d z_{b} f_{0}=N_{b}
$$

where $z_{b}=\theta / k_{r}$ is the internal bunch position and $N_{b}$ is the total number of electrons. To consider a specific case, we select a distribution function of the form

$$
\begin{aligned}
f_{0}= & \frac{N_{b} / l_{b}}{(2 \pi)^{5 / 2} \sigma_{x} \sigma_{y} \sigma_{x}^{\prime} \sigma_{y}^{\prime} \sigma_{\delta}} \exp \left(-\frac{\delta^{2}}{2 \sigma_{\delta}^{2}}\right) \\
& \times \exp \left(-\frac{\left(p_{x}-p_{c e}(z)\right)^{2}+k_{\beta x}^{2}\left(x-x_{c e}(z)\right)^{2}}{2 \sigma_{x}^{\prime 2}}\right) \\
& \times \exp \left(-\frac{p_{y}^{2}+k_{\beta y}^{2} y^{2}}{2 \sigma_{y}^{\prime 2}}\right),
\end{aligned}
$$

which corresponds to a horizontally "kicked" beam with Gaussian energy and transverse profiles but with a uniform longitudinal profile. The latter can be considered as a limiting case of a flattop current profile in which the bunch length $l_{b}$ is much larger than the slippage length $N_{u} \lambda_{r}$, where $N_{u}$ is the number of undulator periods. Thus, the beam current is $I_{p}=q_{e} c N_{b} / l_{b}$ and we can disregard any $\theta$ dependence $\left(\partial f_{0} / \partial \theta=0\right)$. In the previous equation,

$$
x_{c e}(z)=\left(p_{0} / k_{\beta x}\right) \sin \left(k_{\beta x} z_{0}\right)
$$

and

$$
p_{c e}(z)=p_{0} \cos \left(k_{\beta x} z_{0}\right),
$$

(where $z_{0}=z-z_{e}$ and $z_{e}$ is a constant position offset), are the oscillating, horizontal position and momentum centroids of the beam, defined in terms of a transverse slope amplitude $p_{0}$. When $z_{e}=0$, the beam has zero position offset at the start of the undulator $(z=0)$ but a horizontal momentum offset of $p_{0}$, which can thus represent an initial kick. On the other hand, $\sigma_{\delta}$ is the rms energy spread, $\sigma_{x}, \sigma_{y}$ are the rms transverse beam sizes, and $\sigma_{x}^{\prime}, \sigma_{y}^{\prime}$ are the rms values of the transverse slopes. We stress that no offset is considered in the $y$ direction and that the beam is assumed to be matched to the focusing channel, i.e., $\sigma_{x}^{\prime}=\sigma_{x} k_{\beta x}$ and $\sigma_{y}^{\prime}=\sigma_{y} k_{\beta y}$ (so that the beam sizes are constant).

Given the background distribution, the next step is to analytically invert the linearized Vlasov equation [Eq. (6)] by expressing $\hat{f}_{\nu}$ in terms of $E_{\nu}$. This procedure would follow the lines of the method of integration along unperturbed trajectories. The end result is [13]

$\hat{f}_{\nu}=-\kappa_{1} \frac{\partial f_{0}}{\partial \delta} \int_{0}^{z} d \zeta E_{\nu}\left(x_{+}, y_{+}, \zeta\right) \exp \left(-i \Delta \nu k_{u} \zeta+i \theta^{\prime} \xi\right)$,

where $\xi=\zeta-z, \quad x_{+}=x \cos \left(k_{\beta x} \xi\right)+\left(p_{x} / k_{\beta x}\right) \sin \left(k_{\beta x} \xi\right)$ and $y_{+}=y \cos \left(k_{\beta y} \xi\right)+\left(p_{y} / k_{\beta y}\right) \sin \left(k_{\beta y} \xi\right)$. This solution assumes zero initial density modulation $\left(\hat{f}_{\nu}(z=0)=0\right)$ but does not rely on the particular form of $f_{0}$. Substituting Eq. (12) into the right-hand side of Eq. (5) and making use of Eq. (9), we eventually obtain a self-consistent relation that governs the evolution of the radiation amplitude along the undulator:

$$
\begin{aligned}
& \left(\frac{\partial}{\partial z}+\frac{1}{2 i k_{r}}\left[\frac{\partial^{2}}{\partial x^{2}}+\frac{\partial^{2}}{\partial y^{2}}\right]\right) E_{\nu}(x, y, z) \\
& \quad=\int d p_{x} d p_{y} \int_{0}^{z} d \zeta K_{1}\left(x, y, p_{x}, p_{y}, z, \zeta\right) E_{\nu}\left(x_{+}, y_{+}, \zeta\right),
\end{aligned}
$$

with

$$
\begin{aligned}
& K_{1}\left(x, y, p_{x}, p_{y}, z, \zeta\right) \\
& =-\frac{8 i \rho^{3} k_{u}^{3}}{2 \pi \sigma_{x}^{\prime} \sigma_{y}^{\prime}} \xi e^{-i \Delta \nu k_{u} \xi} e^{-2 \sigma_{\delta}^{2} k_{u}^{2} \xi^{2}} \\
& \quad \times \exp \left(-\frac{\left[p_{x}-p_{c e}(z)\right]^{2}+k_{\beta x}^{2}\left[x-x_{c e}(z)\right]^{2}}{2 \sigma_{x}^{\prime 2}}\right) \\
& \quad \times \exp \left(-\frac{p_{y}^{2}+k_{\beta y}^{2} y^{2}}{2 \sigma_{y}^{\prime 2}}\right) \\
& \quad \times \exp \left(-\frac{i k_{r} \xi}{2}\left(p_{x}^{2}+p_{y}^{2}+k_{\beta x}^{2} x^{2}+k_{\beta y}^{2} y^{2}\right)\right) .
\end{aligned}
$$

In the above results,

$$
\rho=\left(\frac{K_{0}^{2}[J J]^{2}}{16 \gamma_{0}^{3} k_{u}^{2} \sigma_{x} \sigma_{y}} \frac{I_{p}}{I_{A}}\right)^{1 / 3}
$$


is the dimensionless FEL (or Pierce) parameter [14] ( $I_{A} \approx 17 \mathrm{kA}$ is the Alfven current). As is well known, the $\rho$ parameter can be viewed as a measure of the frequency bandwidth of the FEL effect.

\section{B. Orthogonal mode expansion}

Having determined the equation that is satisfied by the radiation field, our main goal now is to obtain a solution to Eq. (13) that is compatible with a given input amplitude $E_{\nu}(\mathbf{x}, 0)$ at the entrance of the undulator. To this end, we shall employ an orthogonal expansion technique, relying on a version which takes into account the lack of axial symmetry of the problem at hand. To begin with, we introduce a set of orthogonal transverse modes given by $\psi_{m n}(\mathbf{x}, z)=\chi_{m}(x, z) \varphi_{n}(y, z)$, where

$$
\begin{aligned}
& \chi_{m}(x, z)=\left(2^{m} m !\right)^{-1 / 2} H_{m}\left(\frac{\sqrt{2} \Delta x}{w_{x}}\right) e^{-i m u_{x}} \chi_{0}(x, z), \\
& \varphi_{n}(y, z)=\left(2^{n} n !\right)^{-1 / 2} H_{n}\left(\frac{\sqrt{2} y}{w_{y}}\right) e^{-i n u_{y}} \varphi_{0}(y, z)
\end{aligned}
$$

and

$$
\begin{aligned}
\chi_{0}(x, z)= & \left(k_{r} \beta_{x r} / \pi\right)^{1 / 4}\left(\beta_{x}+i z\right)^{-1 / 2} e^{-k_{r} \alpha_{x i}^{2} /\left(2 \beta_{x r}\right)} \\
& \times \exp \left(-\frac{k_{r}\left(x-\alpha_{x}\right)^{2}}{2\left(\beta_{x}+i z\right)}\right) \\
\varphi_{0}(y, z)= & \left(k_{r} \beta_{y r} / \pi\right)^{1 / 4}\left(\beta_{y}+i z\right)^{-1 / 2} \\
& \times \exp \left(-\frac{k_{r} y^{2}}{2\left(\beta_{y}+i z\right)}\right) .
\end{aligned}
$$

In the above definitions, $m, n=0,1,2,3, \ldots, H_{k}$ are the Hermite polynomials while $\beta_{x}, \beta_{y}$, and $\alpha_{x}$ are complexvalued, $z$-dependent basis parameters. The use of two different basis parameters in $x$ and $y\left(\beta_{x}\right.$ and $\left.\beta_{y}\right)$ accounts for the asymmetry of the problem while the additional parameter $\alpha_{x}$ is introduced in order to deal with the off-axis characteristics of the radiation propagation (which we will verify in due course). The convention according to which an index $r / i$ denotes the real/imaginary part is adopted for these, as well as other, complex variables in this discussion. Thus, the real parts of the three basis functions are denoted by $\beta_{x r}, \beta_{y r}$, and $\alpha_{x r}$, respectively, while their imaginary parts are $\beta_{x i}, \beta_{y i}$, and $\alpha_{x i}$. From a physical point of view, $\beta_{x r}$ and $\beta_{y r}$ represent the local Rayleigh lengths of the radiation while $-\beta_{x i}$ and $-\beta_{y i}$ correspond to the local waist positions. As far as $\alpha_{x}$ is concerned, we will verify that $\alpha_{x i}$ is proportional to the offset of the radiation profile from the origin of the inverse (angle) space while both $\alpha_{x r}$ and $\alpha_{x i}$ contribute to the offset in real space. Moreover,

$$
\begin{aligned}
& w_{x}=\left(\frac{2}{k_{r} \beta_{x r}}\right)^{1 / 2}\left|\beta_{x}+i z\right|, \\
& w_{y}=\left(\frac{2}{k_{r} \beta_{y r}}\right)^{1 / 2}\left|\beta_{y}+i z\right|
\end{aligned}
$$

and

$$
\begin{aligned}
& u_{x}=\arctan \left(\frac{z+\beta_{x i}}{\beta_{x r}}\right), \\
& u_{y}=\arctan \left(\frac{z+\beta_{y i}}{\beta_{y r}}\right)
\end{aligned}
$$

are the waist and Gouy phaselike parameters while $\Delta x=x-x_{c}$, with

$$
x_{c}=\alpha_{x r}+\alpha_{x i} \frac{z+\beta_{x i}}{\beta_{x r}}
$$

representing the centroid of the radiation profile. These modes, which have also been used in the study of an FEL based on a transverse gradient undulator $[13,15]$, satisfy the orthonormality condition

$$
\int d^{2} \mathbf{x} \psi_{m^{\prime} n^{\prime}}^{*}(\mathbf{x}, z) \psi_{m n}(\mathbf{x}, z)=\delta_{m m^{\prime}} \delta_{n n^{\prime}}
$$

and form a complete set. Thus, the radiation amplitude $E_{\nu}(\mathbf{x}, z)$ can (generally speaking) be expanded in terms of the generalized Gauss-Hermite modes described above. Were such an expansion to be substituted back into Eq. (13), the result would be an infinite set of evolution equations for the mode coefficients. However, pursuing a general multimode analysis here is not deemed advisable due to the significantly increased algebraic complexity of the expressions involved, which makes the final analytical results too unwieldy to be of much practical use. Instead, we shall confine our objective to the construction of an approximate solution for the initial value problem which retains only the fundamental ( $m=n=0$ or 00 ) mode. This allows for reasonably compact results without much loss of accuracy in cases where multimode effects are not important in describing the radiation.

In the single-mode approximation, we assume that $E_{\nu}(\mathbf{x}, z) \approx E_{\nu}^{00}(\mathbf{x}, z)=\hat{c}_{00} C_{00}(z) \psi_{00}(\mathbf{x}, z)$, where $\hat{c}_{00}$ is a constant and $C_{00}(z)$ is a dimensionless mode coefficient to be determined along with the basis parameters $\beta_{x}(z)$, $\alpha_{x}(z)$, and $\beta_{y}(z)$ following a self-consistent (or sourcedependent) approach $[16,17]$. Let us also abbreviate Eq. (13) as $\mathcal{T}_{0} E_{\nu}(\mathbf{x}, z)=0$, where $\mathcal{T}_{0}$ is the corresponding integral-differential operator. Since $E_{\nu}^{00}(\mathbf{x}, z)$ is not an exact solution of the amplitude equation, $\mathcal{T}_{0} E_{\nu}^{00}(\mathbf{x}, z)$ is a nonzero function. Instead, our approximate solution is based on imposing the condition that the projections of $\mathcal{T}_{0} E_{\nu}^{00}$ with respect to the $00,10,20$, and 02 modes are zero, i.e.,

$$
\begin{aligned}
\left\langle\psi_{00} \mid \mathcal{T}_{0} E_{\nu}^{00}\right\rangle & =\left\langle\psi_{10} \mid \mathcal{T}_{0} E_{\nu}^{00}\right\rangle \\
& =\left\langle\psi_{20} \mid \mathcal{T}_{0} E_{\nu}^{00}\right\rangle \\
& =\left\langle\psi_{02} \mid \mathcal{T}_{0} E_{\nu}^{00}\right\rangle=0,
\end{aligned}
$$


where we recall that the definition of the inner product is $\langle f \mid g\rangle=\int d^{2} \mathbf{x} f^{*} g$. The projections with respect to the 01 and 11 modes can be readily shown to be identically equal to zero. Utilizing Eqs. (16)-(17), along with the aforementioned conditions, we can obtain a set of four integrodifferential equations for $C_{00}, \beta_{x}, \alpha_{x}$, and $\beta_{y}$. In order to cast these analytical results in a more useful form, we first introduce the scaled variables $\bar{z}=z / \beta_{e x}, \hat{\beta}_{x}=\beta_{x} / \beta_{e x}$, $\hat{\alpha}_{x}=\alpha_{x} / \sigma_{x}$, and $\hat{\beta}_{y}=\beta_{y} / \beta_{e y}$, where $\beta_{e x}=\sigma_{x} / \sigma_{x}^{\prime}=$ $1 / k_{\beta x}$ is the horizontal matched electron beta function and $\beta_{e y}=\sigma_{y} / \sigma_{y}^{\prime}=1 / k_{\beta y}$ is its vertical counterpart. The resulting equations are

$$
\begin{aligned}
\frac{d C_{00}}{d \bar{z}}= & i\left\{\frac{1}{4 \hat{\beta}_{x r}} \frac{d \hat{\beta}_{x i}}{d \bar{z}}+\frac{1}{4 \hat{\beta}_{y r}} \frac{d \hat{\beta}_{y i}}{d \bar{z}}+\hat{B}_{x} \frac{\hat{\alpha}_{x i}}{\hat{\beta}_{x r}}\right. \\
& \left.\times\left(\frac{d \hat{\alpha}_{x r}}{d \bar{z}}+\frac{\hat{\alpha}_{x i}}{2 \hat{\beta}_{x r}} \frac{d \hat{\beta}_{x i}}{d \bar{z}}\right)\right\} C_{00}(\bar{z}) \\
& +\int_{0}^{\bar{z}} d \bar{\zeta} C_{00}(\bar{\zeta}) L_{1}\left(\bar{z}, \bar{\zeta}, \hat{\beta}_{x}, \hat{\beta}_{x, \zeta}, \hat{\alpha}_{x}, \hat{\alpha}_{x, \zeta}, \hat{\beta}_{y}, \hat{\beta}_{y, \zeta}\right),
\end{aligned}
$$

$$
\begin{aligned}
\frac{d \hat{\alpha}_{x}}{d \bar{z}}= & i \frac{\hat{\alpha}_{x i}}{\hat{\beta}_{x r}} \frac{d \hat{\beta}_{x}}{d \bar{z}}+C_{00}^{-1}(\bar{z}) \\
& \times \int_{0}^{\bar{z}} d \bar{\zeta} C_{00}(\bar{\zeta}) L_{2}\left(\bar{z}, \bar{\zeta}, \hat{\beta}_{x}, \hat{\beta}_{x, \zeta}, \hat{\alpha}_{x}, \hat{\alpha}_{x, \zeta}, \hat{\beta}_{y}, \hat{\beta}_{y, \zeta}\right),
\end{aligned}
$$

$\frac{d \hat{\beta}_{x}}{d \bar{z}}=\frac{\int_{0}^{\bar{z}} d \bar{\zeta} C_{00}(\bar{\zeta}) L_{3}\left(\bar{z}, \bar{\zeta}, \hat{\beta}_{x}, \hat{\beta}_{x, \zeta}, \hat{\alpha}_{x}, \hat{\alpha}_{x, \zeta}, \hat{\beta}_{y}, \hat{\beta}_{y, \zeta}\right)}{C_{00}(\bar{z})}$,

and

$\frac{d \hat{\beta}_{y}}{d \bar{z}}=\frac{\int_{0}^{\bar{z}} d \bar{\zeta} C_{00}(\bar{\zeta}) L_{4}\left(\bar{z}, \bar{\zeta}, \hat{\beta}_{x}, \hat{\beta}_{x, \zeta}, \hat{\alpha}_{x}, \hat{\alpha}_{x, \zeta}, \hat{\beta}_{y}, \hat{\beta}_{y, \zeta}\right)}{C_{00}(\bar{z})}$.

In the above equations (and elsewhere), an extra subscript $\zeta$ assigned to a variable denotes a dependence on the integration variable $\bar{\zeta}$, i.e., $\hat{\beta}_{x, \zeta}=\hat{\beta}_{x}(\bar{\zeta}), \hat{\alpha}_{x, \zeta}=\hat{\alpha}_{x}(\bar{\zeta})$, and $\hat{\beta}_{y, \zeta}=\hat{\beta}_{y}(\bar{\zeta})$ etc. The functions $L_{1}-L_{4}$ are given by

$$
\begin{aligned}
L_{1}= & -8 i \hat{A}_{x}^{3 \bar{\xi}} \exp \left(-2 i \hat{A}_{x} \hat{\nu} \bar{\xi}-2 \hat{A}_{x}^{2}\left(\hat{\sigma}_{\delta}\right)^{2} \bar{\xi}^{2}\right) \\
& \times\left(\hat{\beta}_{x r, \zeta} / \hat{\beta}_{x r}\right)^{1 / 4}\left(\hat{\beta}_{y r, \zeta} / \hat{\beta}_{y r}\right)^{1 / 4}\left(\hat{\bar{\beta}}_{x} / \hat{\bar{\beta}}_{x, \zeta}\right)^{1 / 2} \\
& \times\left(\hat{\bar{\beta}}_{y} / \hat{\bar{\beta}}_{y, \zeta}\right)^{1 / 2} G_{p p}^{-1 / 2} \hat{T}_{2}^{-1 / 2} \exp \left(\hat{T}_{0}\right) C_{p p}^{-1 / 2} \hat{Y}_{2}^{-1 / 2}, \\
L_{2}= & \left(\hat{\beta}_{x r} / \hat{B}_{x}\right)^{1 / 2} e^{i u_{x}}\left(\hat{T}_{1} / \hat{T}_{2}\right) L_{1}, \\
L_{3}= & 2 \hat{\beta}_{x r} e^{2 i u_{x}}\left[\hat{T}_{2}^{-1}\left(1+\hat{T}_{1}^{2} /\left(2 \hat{T}_{2}\right)\right)-1\right] L_{1}, \\
L_{4}= & 2 \hat{\beta}_{y r} e^{2 i u_{y}}\left(\hat{Y}_{2}^{-1}-1\right) L_{1},
\end{aligned}
$$

where $\hat{A}_{x}=\rho k_{u} \beta_{e x}, \bar{\xi}=\bar{\zeta}-\bar{z}, \hat{\nu}=\Delta \nu /(2 \rho), \hat{\sigma}_{\delta}=\sigma_{\delta} / \rho$, $\hat{\bar{\beta}}_{x}=\hat{\beta}_{x}+i \bar{z}, \hat{\bar{\beta}}_{y}=\hat{\beta}_{y}+i\left(\beta_{e x} / \beta_{e y}\right) \bar{z}$, and $\hat{B}_{x}=k_{r} \sigma_{x} \sigma_{x}^{\prime}$. From a physical point of view, $\hat{A}_{x}$ is proportional to the ratio of the horizontal beta function to the gain length while $\hat{B}_{x}$ is proportional to the ratio of the horizontal beam emittance $\sigma_{x} \sigma_{x}^{\prime}$ to the radiation emittance $\lambda_{r} / 4 \pi$. Furthermore, $\quad \hat{T}_{1}=G_{\theta}+G_{\theta p} G_{p} / G_{p p}, \quad \hat{T}_{2}=(1 / 2)$ $\left(G_{\theta \theta}-G_{\theta p}^{2} / G_{p p}\right), \hat{Y}_{2}=(1 / 2)\left(C_{t t}-C_{t p}^{2} / C_{p p}\right)$, and

$$
\begin{aligned}
\hat{T}_{0}= & \frac{G_{p}^{2}}{2 G_{p p}}+\frac{\hat{T}_{1}^{2}}{4 \hat{T}_{2}}-\frac{1}{2}\left(1+i \hat{B}_{x} \bar{\xi}\right) \hat{x}_{c}^{2}+\hat{p}_{0} \hat{x}_{c} \sin \bar{z}_{0}-\frac{\hat{p}_{0}^{2}}{2} \\
& -\frac{\hat{B}_{x}}{2}\left\{\frac{\hat{\alpha}_{x i}^{2}}{\hat{\beta}_{x r}}+\frac{\hat{\alpha}_{x i, \zeta}^{2}}{\hat{\beta}_{x r, \zeta}}+\frac{\left(\hat{x}_{c}-\hat{\alpha}_{x}^{*}\right)^{2}}{\hat{\beta}_{x}^{*}}+\frac{\left(\hat{x}_{c} \cos \bar{\xi}-\hat{\alpha}_{x, \zeta}\right)^{2}}{\hat{\beta}_{x, \zeta}}\right\},
\end{aligned}
$$

where $\bar{z}_{0}=z_{0} / \beta_{e x}, \hat{x}_{c}=x_{c} / \sigma_{x}=\hat{\alpha}_{x r}+\hat{\alpha}_{x i}\left(\hat{z}+\hat{\beta}_{x i}\right) / \hat{\beta}_{x r}$, and $\hat{p}_{0}=p_{0} / \sigma_{x}^{\prime}$. The remaining quantities to be defined are

$$
\begin{aligned}
& G_{p p}=1+i \hat{B}_{x} \bar{\xi}+\frac{\hat{B}_{x} \sin ^{2} \bar{\xi}}{\hat{\bar{\beta}}_{x, \zeta}}, \\
& G_{\theta \theta}=\hat{\bar{\beta}}_{x} / \hat{\beta}_{x r}+\tilde{w}_{x}^{2}\left(\cos ^{2} \bar{\xi} / \hat{\bar{\beta}}_{x, \zeta}+1 / \hat{B}_{x}+i \bar{\xi}\right), \\
& G_{\theta p}=-\frac{\hat{B}_{x}{ }^{1 / 2}}{\hat{\bar{\beta}}_{x, \zeta}} \tilde{w}_{x} \cos \bar{\xi} \sin \bar{\xi}, \\
& G_{p}=\hat{p}_{0} \cos \bar{z}_{0}-\frac{\hat{B}_{x}}{\hat{\bar{\beta}}_{x, \zeta}}\left(\hat{x}_{c} \cos \bar{\xi}-\hat{\alpha}_{x, \zeta}\right) \sin \bar{\xi}, \\
& G_{\theta}=-\frac{\hat{B}_{x}^{1 / 2}}{\hat{\bar{\beta}}_{x}^{*}} \tilde{w}_{x}\left(\hat{x}_{c}-\hat{\alpha}_{x}^{*}\right)-\left(1+i \hat{B}_{x} \bar{\xi}\right) \frac{\hat{x}_{c} \tilde{w}_{x}}{\hat{B}_{x}^{1 / 2}} \\
& -\frac{\hat{B}_{x}^{1 / 2}}{\hat{\bar{\beta}}_{x, \zeta}} \tilde{w}_{x} \cos \bar{\xi}\left(\hat{x}_{c} \cos \bar{\xi}-\hat{\alpha}_{x, \zeta}\right)+\hat{p}_{0} \frac{\tilde{w}_{x} \sin \bar{z}_{0}}{\hat{B}_{x}^{1 / 2}},
\end{aligned}
$$

where $\tilde{w}_{x}=\left|\hat{\bar{\beta}}_{x}\right| / \hat{\beta}_{x r}^{1 / 2}$, and

$$
\begin{aligned}
C_{p p} & =1+i \hat{B}_{y} \tilde{\xi}+\hat{B}_{y} \sin ^{2} \tilde{\xi} / \hat{\bar{\beta}}_{y, \zeta}, \\
C_{t t} & =\hat{\bar{\beta}}_{y} / \hat{\beta}_{y r}+\tilde{w}_{y}^{2}\left[\hat{B}_{y}^{-1}+i \tilde{\xi}+\cos ^{2} \tilde{\xi} / \hat{\bar{\beta}}_{y, \zeta}\right], \\
C_{t p} & =-\hat{B}_{y}^{1 / 2}\left(\tilde{w}_{y} / \hat{\bar{\beta}}_{y, \zeta}\right) \cos \tilde{\xi} \sin \tilde{\xi},
\end{aligned}
$$

with $\hat{B}_{y}=k_{r} \sigma_{y} \sigma_{y}^{\prime}, \quad \tilde{\xi}=\left(\beta_{e x} / \beta_{e y}\right) \bar{\xi}$, and $\tilde{w}_{y}=\left|\hat{\bar{\beta}}_{y}\right| / \hat{\beta}_{y r}^{1 / 2}$. Given the values of $C_{00}, \beta_{x}, \beta_{y}$, and $\alpha_{x}$ at $z=0$, numerically solving Eqs. (23)-(26) yields an approximate solution to the 3D initial value problem of the FEL. This solution can be used to provide a quantitative description of the FEL radiation in the linear regime. The radiation power $P_{r}$-which is defined by $P_{r}=\int d^{2} \mathbf{x} I_{r}(\mathbf{x}, z)$, where $I_{r}=$ $\left(c \varepsilon_{0} / 2\right)\left|E_{\nu}\right|^{2}$ is the radiation intensity (and $\varepsilon_{0}$ is the vacuum permittivity) - is given by 


$$
P_{r}=P_{0}\left|C_{00}\right|^{2},
$$

where the initial condition for the mode coefficient is assumed to be $C_{00}(0)=1$ and $P_{0}=c \varepsilon_{0}\left|\hat{c}_{00}\right|^{2} / 2$ is the input radiation power. Moreover, the rms radiation beam sizes are, in turn, given by

$$
\sigma_{r x}=\sqrt{\left\langle x^{2}\right\rangle_{r}-\langle x\rangle_{r}^{2}}=w_{x} / 2
$$

and

$$
\sigma_{r y}=\sqrt{\left\langle y^{2}\right\rangle_{r}}=w_{y} / 2,
$$

where $\langle x\rangle_{r}=x_{c}$ and the $\langle\ldots\rangle_{r}$ average uses the spatial intensity $I_{r}$ as a weighting function [for example, we have $\left\langle x^{2}\right\rangle_{r}=\int d^{2} \mathbf{x} x^{2} I_{r}(\mathbf{x}, z) / \int d^{2} \mathbf{x} I_{r}(\mathbf{x}, z)$ etc.]. As far as the situation in the inverse transverse (i.e. momentum) space is concerned, an inspection of the Fourier transform definition $\tilde{E}_{\nu}(\mathbf{k}, z) \propto \int d^{2} \mathbf{x} E_{\nu}(\mathbf{x}, z) \exp (i \mathbf{k} \mathbf{x})$ leads to a Gaussian angular profile with

$$
\left|\tilde{E}_{\nu}(\mathbf{k}, z)\right|^{2} \propto \exp \left(-\frac{\left(k_{x}-k_{0}\right)^{2}}{2 \sigma_{k x}^{2}}\right) \exp \left(-\frac{k_{y}^{2}}{2 \sigma_{k y}^{2}}\right),
$$

where

$$
\begin{aligned}
\sigma_{k x}^{2} & =k_{r}^{2} \sigma_{r x}^{\prime}{ }^{2}=k_{r} /\left(2 \beta_{x r}\right), \\
\sigma_{k y}^{2} & =k_{r}^{2} \sigma_{r y}^{\prime 2}=k_{r} /\left(2 \beta_{y r}\right), \\
k_{0} & =k_{r} x_{c}^{\prime}=-k_{r}\left(\alpha_{x i} / \beta_{x r}\right) .
\end{aligned}
$$

In the above equations, $\sigma_{r x}^{\prime}$ and $\sigma_{r y}^{\prime}$ are the rms angular sizes while $x_{c}^{\prime}$ is the angular centroid of the radiation.

Thus far, we have only considered properties of the radiation. As far as the electron beam is concerned, the perturbation formalism we have been using also allows us to calculate a quantitative expression (valid in the linear regime) for the bunching that is induced by the FEL effect. The bunching factor of the electron beam can be defined in terms of the distribution function $f$ as the absolute value of the average $\left\langle e^{-i \theta}\right\rangle$, which is in turn defined by

$$
\left\langle e^{-i \theta}\right\rangle=\frac{\int d^{2} \mathbf{p} \int d^{2} \mathbf{x} \int d \delta \int_{0}^{2 \pi} d \theta f e^{-i \theta}}{\int d^{2} \mathbf{p} \int d^{2} \mathbf{x} \int d \delta \int_{0}^{2 \pi} d \theta f},
$$

where $\mathbf{p}=\left(p_{x}, p_{y}\right)$. Recalling that $f=f_{0}+f_{1}$, with $f_{0}$ being $\theta$ independent and normalized according to Eq. (8) while $f_{1}$ has a sinusoidal modulation with $\theta$ according to $f_{1} \approx \hat{f}_{\nu} e^{i \theta}+$ c.c. $(\nu \approx 1)$, the expression for the phase average reduces to

$$
\left\langle e^{-i \theta}\right\rangle=\frac{2 \pi \int d^{2} \mathbf{p} \int d^{2} \mathbf{x} \int d \delta \hat{f}_{\nu}}{2 \pi\left(N_{b} / l_{b}\right)} .
$$

In view of Eq. (5), Eq. (36) becomes

$$
\left\langle e^{-i \theta}\right\rangle=-\frac{l_{b}}{N_{b}} \frac{1}{\kappa_{2}} e^{-i \Delta \nu k_{u} z} \int d^{2} \mathbf{x} L E_{\nu}(\mathbf{x}, z),
$$

where $L=\partial / \partial z+\nabla_{\perp}^{2} /\left(2 i k_{r}\right)$ is the paraxial operator. Making use of the assumption that $E_{\nu}(\mathbf{x}, z) \approx$ $\hat{c}_{00} C_{00}(z) \psi_{00}(\mathbf{x}, z)$ (i.e. the single mode, Gaussian approximation adopted earlier) in conjunction with Eqs. (17) and (37), we find-after some algebra - that the bunching factor is given by

$$
\begin{aligned}
\left|\left\langle e^{-i \theta}\right\rangle\right|= & \frac{l_{b}}{N_{b} \kappa_{2}}\left(\frac{2 P_{r}}{c \varepsilon_{0}}\right)^{1 / 2}\left(\frac{4 \pi}{k_{r}}\right)^{1 / 2} \beta_{x r}^{1 / 4} \beta_{y r}^{1 / 4} \\
& \times\left|i \mu_{z}+\frac{1}{4 \beta_{x r}} \frac{d \beta_{x r}}{d z}+\frac{1}{4 \beta_{y r}} \frac{d \beta_{y r}}{d z}-\frac{d Q_{E}}{d z}\right| e^{-Q_{E},}
\end{aligned}
$$

where $Q_{E}=k_{r} \alpha_{x i}^{2} /\left(2 \beta_{x r}\right)$ and $\mu_{z}$ is a $z$-dependent, local complex growth rate that is defined through the relation $C_{00}(z)=\exp \left(i \int_{0}^{z} d s \mu_{z}(s)\right)$ or, equivalently,

$$
\mu_{z}(z)=-\frac{i}{C_{00}(z)} \frac{d C_{00}(z)}{d z} .
$$

A more convenient expression can be obtained if one switches to the scaled variables discussed earlier. In terms of that scaling, we find that the expression for the bunching factor is

$$
\begin{aligned}
\left|\left\langle e^{-i \theta}\right\rangle\right|= & \left(\frac{2 P_{r}}{P_{S}}\right)^{1 / 2}\left(\frac{\hat{\beta}_{x r}}{\hat{B}_{x}}\right)^{1 / 4}\left(\frac{\hat{\beta}_{y r}}{\hat{B}_{y}}\right)^{1 / 4} \\
& \times\left|i \hat{\mu}_{z}+\frac{1}{2 \hat{A}_{x}}\left[\frac{1}{4 \hat{\beta}_{x r}} \frac{d \hat{\beta}_{x r}}{d \bar{z}}+\frac{1}{4 \hat{\beta}_{y r}} \frac{d \hat{\beta}_{y r}}{d \bar{z}}-\frac{d Q_{E}}{d \bar{z}}\right]\right| e^{-Q_{E}},
\end{aligned}
$$

where $P_{S}=\rho \gamma_{0} m_{e} c^{2} I_{p} / q_{e}, Q_{E}=\hat{B}_{x} \hat{\alpha}_{x i}^{2} /\left(2 \hat{\beta}_{x r}\right)$, and $\hat{\mu}_{z}=$ $\mu_{z} /\left(2 \rho k_{u}\right)$, all other symbols having already been defined. Taking into account the fact that $\left|\left\langle e^{-i \theta}\right\rangle\right| \leq 1$, as well as the observation that the part on the right-hand side of the above equation which contains the various scaled basis parameters is (generally speaking) of the order of unity, it follows that the perturbation result given in Eq. (40) should break down when $P_{r} \sim P_{S}=\rho P_{\text {beam }}$, where $P_{\text {beam }}=\gamma_{0} m_{e} c^{2} I_{p} / q_{e}$ is the $e$-beam power. Viewed as a qualitative limit for the applicability of the linearized model, this condition reproduces the established fact that the saturation power is of the order of the $e$-beam power multiplied by the FEL parameter.

\section{Periodic analysis}

In the previous section, we described a technique for obtaining a semianalytical, approximate solution to the initial 
value problem of the FEL. That solution can adequately describe the FEL interaction in the linear regime, given the initial conditions. However, it is also possible to develop a method similar in form to the standard FEL eigenmode approach [18], which would lead to a description independent of initial conditions. This is made possible by exploiting the periodic $z$ dependence that is introduced by the centroid oscillation. Starting from the radiation amplitude equation [Eq. (13)], our first step is to switch to the new horizontal variables $\bar{p}_{x}=p_{x}-p_{c e}(z)$ and $\bar{x}=x-x_{c e}(z)$. This manipulation allows us to utilize a frame that is comoving with the $e$-beam. Its end result is the equation

$$
\begin{gathered}
\left(\frac{\partial}{\partial z}-p_{0} \cos \left(k_{\beta x} z_{0}\right) \frac{\partial}{\partial \bar{x}}+\frac{1}{2 i k_{r}}\left[\frac{\partial^{2}}{\partial \bar{x}^{2}}+\frac{\partial^{2}}{\partial y^{2}}\right]\right) \bar{E}_{\nu}(\bar{x}, y, z) \\
=\int d \bar{p}_{x} d p_{y} \int_{0}^{z} d \zeta K_{2}\left(\bar{x}, y, \bar{p}_{x}, p_{y}, z, \zeta\right) \bar{E}_{\nu}\left(\bar{x}_{+}, y_{+}, \zeta\right),
\end{gathered}
$$

where $\bar{E}_{\nu}(\bar{x}, y, z)=E_{\nu}\left(\bar{x}+x_{c e}(z), y, z\right)$ is a new amplitude quantity, $\bar{x}_{+}=\bar{x} \cos \left(k_{\beta x} \xi\right)+\left(\bar{p}_{x} / k_{\beta x}\right) \sin \left(k_{\beta x} \xi\right)$, and

$$
\begin{aligned}
K_{2}\left(\bar{x}, y, \bar{p}_{x}, p_{y}, z, \zeta\right) \\
=-\frac{8 i \rho^{3} k_{u}^{3}}{2 \pi \sigma_{x}^{\prime} \sigma_{y}^{\prime}} \xi e^{-i \Delta \nu_{s} k_{u} \xi} e^{-2 \sigma_{\delta}^{2} k_{u}^{2} \xi^{2}} \\
\quad \times \exp \left(-\frac{\bar{p}_{x}^{2}+k_{\beta x}^{2} \bar{x}^{2}}{2 \sigma_{x}^{\prime 2}}-\frac{p_{y}^{2}+k_{\beta y}^{2} y^{2}}{2 \sigma_{y}^{\prime 2}}\right) \\
\quad \times \exp \left(-\frac{i k_{r} \xi}{2}\left(\bar{p}_{x}^{2}+p_{y}^{2}+k_{\beta x}^{2} \bar{x}^{2}+k_{\beta y}^{2} y^{2}\right)\right) \\
\quad \times \exp \left\{-i p_{0} k_{r} \xi\left[\bar{p}_{x} \cos \left(k_{\beta x} z_{0}\right)+k_{\beta x} \bar{x} \sin \left(k_{\beta x} z_{0}\right)\right]\right\} .
\end{aligned}
$$

In the above equation, we have introduced the shifted detuning $\Delta \nu_{s}$, which is defined through the relation

$$
\Delta \nu_{s} k_{u}=\Delta \nu k_{u}+\left(k_{r} / 2\right) p_{0}^{2} .
$$

This definition is consistent with the increase in the FEL wavelength due to the kick. To proceed, we need to define some additional scaled quantities, starting with the following: $\hat{x}=\bar{x} / \sigma_{x}, \hat{y}=y / \sigma_{y}, \hat{p}_{x}=\bar{p}_{x} / \sigma_{x}^{\prime}, \hat{p}_{y}=$ $p_{y} / \sigma_{y}^{\prime}, \quad \hat{z}=2 \rho k_{u} z, \quad \hat{z}_{0}=2 \rho k_{u} z_{0}, \quad \hat{\zeta}=2 \rho k_{u} \zeta, \quad$ and $\hat{\xi}=\hat{\zeta}-\hat{z}=2 \rho k_{u} \xi$. Moreover, we also introduce the scaled detuning $\hat{\nu}_{s}=\Delta \nu_{s} /(2 \rho)$, the scaled energy spread $\hat{\sigma}_{\delta}=\sigma_{\delta} / \rho$, the diffraction parameters $\eta_{d x}=\left(4 k_{u} k_{r} \rho \sigma_{x}^{2}\right)^{-1}$ and $\eta_{d y}=\left(4 k_{u} k_{r} \rho \sigma_{y}^{2}\right)^{-1}$, as well as the focusing/emittance parameters $\hat{k}_{\beta x}=k_{\beta x} /\left(2 \rho k_{u}\right), \quad \hat{k}_{\beta y}=k_{\beta y} /\left(2 \rho k_{u}\right), \quad \eta_{\varepsilon x}=$ $k_{r} \sigma_{x}^{\prime 2} /\left(2 \rho k_{u}\right)$, and $\eta_{\varepsilon y}=k_{r} \sigma_{y}^{\prime 2} /\left(2 \rho k_{u}\right)$. We note that the latter four parameters satisfy the relations $\eta_{\varepsilon x}=\hat{k}_{\beta x}^{2} /\left(2 \eta_{d x}\right)$ and $\eta_{\varepsilon y}=\hat{k}_{\beta y}^{2} /\left(2 \eta_{d y}\right)$. Last but not least, we also define two scaled parameters that are proportional to the kick angle $p_{0}$ :

$$
\epsilon_{1}=p_{0} /\left(2 \rho k_{u} \sigma_{x}\right)
$$

and

$$
\epsilon=k_{r} p_{0} \sigma_{x}^{\prime} /\left(2 \rho k_{u}\right) \text {. }
$$

We note that their ratio is related to the horizontal emittance $\epsilon_{x}=\sigma_{x} \sigma_{x}^{\prime}$ since $\epsilon / \epsilon_{1}=k_{r} \sigma_{x} \sigma_{x}^{\prime}$. The scaled evolution equation is

$$
\begin{gathered}
\left(\frac{\partial}{\partial \hat{z}}-\epsilon_{1} \cos \left(\hat{k}_{\beta x} \hat{z}_{0}\right) \frac{\partial}{\partial \hat{x}}-i\left[\eta_{d x} \frac{\partial^{2}}{\partial \hat{x}^{2}}+\eta_{d y} \frac{\partial^{2}}{\partial \hat{y}^{2}}\right]\right) \hat{E}_{\nu}(\hat{x}, \hat{y}, \hat{z}) \\
=\int d \hat{p}_{x} d \hat{p}_{y} \int_{0}^{\hat{z}} d \hat{\zeta} \hat{K}_{3}\left(\hat{x}, \hat{y}, \hat{p}_{x}, \hat{p}_{y}, \hat{z}, \hat{\zeta}\right) \hat{E}_{\nu}\left(\hat{x}_{+}, \hat{y}_{+}, \hat{\zeta}\right)
\end{gathered}
$$

where $\hat{x}_{+}=\bar{x}_{+} / \sigma_{x}=\hat{x} \cos \left(\hat{k}_{\beta x} \hat{\xi}\right)+\hat{p}_{x} \sin \left(\hat{k}_{\beta x} \hat{\xi}\right), \quad \hat{y}_{+}=$ $y_{+} / \sigma_{y}=\hat{y} \cos \left(\hat{k}_{\beta y} \hat{\xi}\right)+\hat{p}_{y} \sin \left(\hat{k}_{\beta y} \hat{\xi}\right)$ and

$$
\begin{aligned}
\hat{K}_{3}\left(\hat{x}, \hat{y}, \hat{p}_{x}, \hat{p}_{y}, \hat{z}, \hat{\zeta}\right) \\
=-\frac{i \hat{\xi}}{2 \pi} \exp \left(-i \hat{\nu}_{s} \hat{\xi}-\hat{\sigma}_{\delta}^{2} \hat{\xi}^{2} / 2\right) \\
\quad \times \exp \left(-\frac{1+i \eta_{\varepsilon x} \hat{\xi}}{2}\left(\hat{p}_{x}^{2}+\hat{x}^{2}\right)-\frac{1+i \eta_{\varepsilon y} \hat{\xi}}{2}\left(\hat{p}_{y}^{2}+\hat{y}^{2}\right)\right) \\
\quad \times \exp \left(-i \epsilon \hat{\xi}\left[\hat{p}_{x} \cos \left(\hat{k}_{\beta x} \hat{z}_{0}\right)+\hat{x} \sin \left(\hat{k}_{\beta x} \hat{z}_{0}\right)\right]\right) .
\end{aligned}
$$

The term proportional to $\partial / \partial \hat{x}$ in the first line of Eq. (46) and the entire third line of Eq. (47) are the new features introduced by $p_{0}$. The latter also contains an explicit, periodic dependence on $\hat{z}_{0}$ (i.e. not one implicitly contained in $\hat{\xi}=\hat{\zeta}-\hat{z}$ ). Expanding this exponential term up to second order in $\epsilon$, we have

$$
\begin{aligned}
Q_{P}= & \exp \left(-i \epsilon \hat{\xi}\left[\hat{p}_{x} \cos \left(\hat{k}_{\beta x} \hat{z}_{0}\right)+\hat{x} \sin \left(\hat{k}_{\beta x} \hat{z}_{0}\right)\right]\right) \\
\approx & 1-i \epsilon \hat{\xi}\left[\hat{p}_{x} \cos \left(\hat{k}_{\beta x} \hat{z}_{0}\right)+\hat{x} \sin \left(\hat{k}_{\beta x} \hat{z}_{0}\right)\right] \\
& -\frac{\epsilon^{2} \hat{\xi}^{2}}{2}\left[\hat{p}_{x}^{2} \cos ^{2}\left(\hat{k}_{\beta x} \hat{z}_{0}\right)+\hat{x}^{2} \sin ^{2}\left(\hat{k}_{\beta x} \hat{z}_{0}\right)\right. \\
& \left.+\hat{x} \hat{p}_{x} \sin \left(2 \hat{k}_{\beta x} \hat{z}_{0}\right)\right]+\cdots
\end{aligned}
$$

so the coefficients in the Fourier series

$$
\begin{aligned}
Q_{P}= & \hat{d}_{0}+\hat{d}_{1} e^{i \hat{k}_{\beta x} \hat{z}_{0}}+\hat{d}_{-1} e^{-i \hat{k}_{\beta x} \hat{z}_{0}} \\
& +\hat{d}_{2} e^{2 i \hat{k}_{\beta x} \hat{z}_{0}}+\hat{d}_{-2} e^{-2 i \hat{k}_{\beta x} \hat{z}_{0}}+\cdots
\end{aligned}
$$

are given by 


$$
\begin{aligned}
\hat{d}_{0}\left(\hat{x}, \hat{p}_{x}, \hat{\xi}\right)= & 1-\frac{\epsilon^{2} \hat{\xi}^{2}}{2}\left[\frac{\hat{p}_{x}^{2}+\hat{x}^{2}}{2}\right]+O\left(\epsilon^{4}\right), \\
\hat{d}_{1}\left(\hat{x}, \hat{p}_{x}, \hat{\xi}\right)= & -\hat{d}_{-1}^{*}\left(\hat{x}, \hat{p}_{x}, \hat{\xi}\right)=-\epsilon \hat{\xi} \frac{\hat{x}+i \hat{p}_{x}}{2}+O\left(\epsilon^{3}\right), \\
\hat{d}_{2}\left(\hat{x}, \hat{p}_{x}, \hat{\xi}\right)= & \hat{d}_{-2}^{*}\left(\hat{x}, \hat{p}_{x}, \hat{\xi}\right)=\frac{\epsilon^{2} \hat{\xi}^{2}}{2}\left[\frac{\hat{x}^{2}-\hat{p}_{x}^{2}}{4}+\frac{i}{2} \hat{x} \hat{p}_{x}\right] \\
& +O\left(\epsilon^{4}\right),
\end{aligned}
$$

etc. In a more compact form, one can show that the above Fourier coefficients are also given by $\hat{d}_{n}=$ $J_{-n}\left(\epsilon \hat{\xi} \sqrt{\hat{p}_{x}^{2}+\hat{x}^{2}}\right) e^{i n \theta_{E}}$, where $\theta_{E}=\arctan \left(\hat{p}_{x} / \hat{x}\right)$. This expression is derived using the Jacobi-Anger expansion and is valid even when $\epsilon>1$. However, we will see that the expansion in terms of $\epsilon$ given by Eq. (50) is quite sufficient for our purposes.

In view of the periodic $z$ dependence of the problem at hand, we try a solution of the form

$$
\hat{E}_{\nu}(\hat{x}, \hat{y}, \hat{z})=\sum_{n=-\infty}^{\infty} A_{n}(\hat{x}, \hat{y}) e^{i\left(\hat{\mu}+n \hat{k}_{\beta x}\right) \hat{z}_{0}}
$$

where $A_{n}$ are Fourier amplitudes and $\hat{\mu}$ is a complex growth rate. Alternatively, this solution can be viewed as a transverse profile that is periodic in $\hat{z}_{0}$, multiplied by $\exp \left(i \hat{\mu} \hat{z}_{0}\right)$. Substituting the above ansatz into Eq. (46) yields an infinite set of coupled equations, namely

$$
\begin{aligned}
& \left(\hat{\mu}+n \hat{k}_{\beta x}-\left[\eta_{d x} \frac{\partial^{2}}{\partial \hat{x}^{2}}+\eta_{d y} \frac{\partial^{2}}{\partial \hat{y}^{2}}\right]\right) A_{n}(\hat{x}, \hat{y}) \\
& \quad+\frac{i \epsilon_{1}}{2}\left(\frac{\partial A_{n-1}(\hat{x}, \hat{y})}{\partial \hat{x}}+\frac{\partial A_{n+1}(\hat{x}, \hat{y})}{\partial \hat{x}}\right) \\
& =\sum_{j=-\infty}^{\infty} \int d \hat{x}_{+} d \hat{y}_{+} \int_{-\infty}^{0} d \hat{\xi} \tilde{Q}_{-j}\left(\hat{x}, \hat{y}, \hat{x}_{+}, \hat{y}_{+}, \hat{\xi}\right) \\
& \quad \times A_{n+j}\left(\hat{x}_{+}, \hat{y}_{+}\right) \exp \left(i\left[\hat{\mu}+(n+j) \hat{k}_{\beta x}\right] \hat{\xi}\right) .
\end{aligned}
$$

In the relation given above, we have changed the transverse integration variables from $\left(\hat{p}_{x}, \hat{p}_{y}\right)$ to $\left(\hat{x}_{+}, \hat{y}_{+}\right)$. As we will see later on, this particular choice allows for some very useful insight regarding the structure of the problem. Moreover,

$\tilde{Q}_{j}\left(\hat{x}, \hat{y}, \hat{x}_{+}, \hat{y}_{+}, \hat{\xi}\right)=\tilde{d}_{j}\left(\hat{x}, \hat{x}_{+}, \hat{\xi}\right) \tilde{\Pi}\left(\hat{x}, \hat{y}, \hat{x}_{+}, \hat{y}_{+}, \hat{\xi}\right)$,

where

$$
\begin{aligned}
& \tilde{\Pi}\left(\hat{x}, \hat{y}, \hat{x}_{+}, \hat{y}_{+}, \hat{\xi}\right) \\
& =-\frac{\hat{\xi} \exp \left(-i \hat{\nu}_{s} \hat{\xi}-\hat{\sigma}_{\delta}^{2} \hat{\xi}^{2} / 2\right)}{2 \pi\left|\sin \left(\hat{k}_{\beta x} \hat{\xi}\right) \sin \left(\hat{k}_{\beta y} \hat{\xi}\right)\right|} \\
& \quad \times \exp \left(-\frac{1+i \eta_{\varepsilon x} \hat{\xi}}{2 \sin ^{2}\left(\hat{k}_{\beta x} \hat{\xi}\right)}\left[\hat{x}_{+}^{2}+\hat{x}^{2}-2 \hat{x} \hat{x}_{+} \cos \left(\hat{k}_{\beta x} \hat{\xi}\right)\right]\right) \\
& \quad \times \exp \left(-\frac{1+i \eta_{\varepsilon y} \hat{\xi}}{2 \sin ^{2}\left(\hat{k}_{\beta y} \hat{\xi}\right)}\left[\hat{y}_{+}^{2}+\hat{y}^{2}-2 \hat{y} \hat{y}_{+} \cos \left(\hat{k}_{\beta y} \hat{\xi}\right)\right]\right)
\end{aligned}
$$

and

$$
\begin{aligned}
\tilde{d}_{0}= & 1-\frac{\epsilon^{2} \hat{\xi}^{2}\left[\hat{x}_{+}^{2}+\hat{x}^{2}-2 \hat{x} \hat{x}_{+} \cos \left(\hat{k}_{\beta x} \hat{\xi}\right)\right]}{4 \sin ^{2}\left(\hat{k}_{\beta x} \hat{\xi}\right)}+O\left(\epsilon^{4}\right), \\
\tilde{d}_{ \pm 1}= & \mp \frac{\epsilon \hat{\xi}\left[\left(\sin \left(\hat{k}_{\beta x} \hat{\xi}\right) \mp i \cos \left(\hat{k}_{\beta x} \hat{\xi}\right)\right) \hat{x} \pm i \hat{x}_{+}\right]}{2 \sin \left(\hat{k}_{\beta x} \hat{\xi}\right)} \\
& +O\left(\epsilon^{3}\right)
\end{aligned}
$$

etc. In what follows, we confine our attention to a model that includes only the fundamental amplitude $A_{0}$ and the first two satellite amplitudes $A_{ \pm 1}$. Even with this simplification, the resulting truncated set of coupled amplitude equations is not amenable to analytical treatment. Instead, we follow a perturbation approach, which is valid when $\epsilon$ and $\epsilon_{1}$ are smaller than unity (i.e. when the kick angle $p_{0}$ is sufficiently small). The latter condition can be further clarified by noting that

$$
\epsilon=\left(2 \pi \sqrt{3} / f_{3 D}\right)^{1 / 2} \sqrt{\eta_{\varepsilon x}}\left(p_{0} / \theta_{c}\right)
$$

and

$$
\epsilon_{1}=\left(4 \pi \sqrt{3} / f_{3 D}\right)^{1 / 2} \sqrt{\eta_{d x}}\left(p_{0} / \theta_{c}\right),
$$

where $f_{3 D}>1$ is a dimensionless parameter representing the gain length increase due to transverse effects and energy spread (see Sec. III for more details). Thus, the smallness of $\epsilon, \epsilon_{1}$ can be directly related to the ratio of the kick to the critical angle.

Our main objective is to obtain the average growth rate $\hat{\mu}$ in the form of a series expansion in terms of $p_{0}$. Thus, we assume that $\hat{\mu}=\hat{\mu}^{(0)}+\hat{\mu}^{(1)}+\hat{\mu}^{(2)}+\cdots$-where $\hat{\mu}^{(k)} \sim p_{0}^{k}$ - and also $A_{n}=A_{n}^{(0)}+A_{n}^{(1)}+A_{n}^{(2)}+\cdots$ (for $n=0, \pm 1$ ), seeking to determine the contribution of each order. To implement this approach, we need to expand the integral kernels $\tilde{Q}_{j}$ in terms of $p_{0}$. Apart from Eqs. (50) and (55), we need to take into account the fact that the shifted detuning $\hat{\nu}_{s}$ also depends on the kick angle via $\hat{\nu}_{s}=\hat{\nu}+\epsilon_{2}$, where $\hat{\nu}=\Delta \nu /(2 \rho)$ and $\epsilon_{2} \equiv k_{r} p_{0}^{2} /\left(4 \rho k_{u}\right)=\epsilon_{1}^{2} /\left(4 \eta_{d x}\right)$. For $p_{0}=0, \tilde{Q}_{j}=0$ for all $j \neq 0$ and we only need to 
deal with the fundamental amplitude $A_{0}(\hat{x}, \hat{y})$. In this case, there is no periodic $z$ dependence and our solution reduces to a standard FEL guided mode, which satisfies the zeroth order mode equation

$$
\begin{aligned}
& \left(\hat{\mu}^{(0)}-\left[\eta_{d x} \frac{\partial^{2}}{\partial \hat{x}^{2}}+\eta_{d y} \frac{\partial^{2}}{\partial \hat{y}^{2}}\right]\right) A_{0}^{(0)}(\hat{x}, \hat{y}) \\
& =\int d \hat{x}_{+} d \hat{y}_{+} \int_{-\infty}^{0} d \hat{\xi} \tilde{Q}_{0}^{(0)}\left(\hat{x}, \hat{y}, \hat{x}_{+}, \hat{y}_{+}, \hat{\xi}\right) \\
& \quad \times A_{0}^{(0)}\left(\hat{x}_{+}, \hat{y}_{+}\right) \exp \left(i \hat{\mu}^{(0)} \hat{\xi}\right),
\end{aligned}
$$

where $\tilde{Q}_{0}^{(0)}\left(\hat{x}, \hat{y}, \hat{x}_{+}, \hat{y}_{+}, \hat{\xi}\right)=\tilde{\Pi}\left(\hat{x}, \hat{y}, \hat{x}_{+}, \hat{y}_{+}, \hat{\xi}\right)_{\hat{\nu}_{s} \rightarrow \hat{\nu}}$. This equation is the standard version that has been studied extensively in 3D theory [18]. Its solutions can be determined through variational theory (among other methods). The latter is based on constructing the following functional:

$$
\begin{aligned}
& \int d \hat{x} d \hat{y} A_{0}^{(0)}(\hat{x}, \hat{y})\left\{\hat{\mu}^{(0)}-\left[\eta_{d x} \frac{\partial^{2}}{\partial \hat{x}^{2}}+\eta_{d y} \frac{\partial^{2}}{\partial \hat{y}^{2}}\right]\right\} A_{0}^{(0)}(\hat{x}, \hat{y}) \\
& =\int d \hat{x} d \hat{y} A_{0}^{(0)}(\hat{x}, \hat{y}) \int d \hat{x}_{+} d \hat{y}_{+} \int_{-\infty}^{0} d \hat{\xi} \\
& \quad \times \tilde{Q}_{0}^{(0)}\left(\hat{x}, \hat{y}, \hat{x}_{+}, \hat{y}_{+}, \hat{\xi}\right) A_{0}^{(0)}\left(\hat{x}_{+}, \hat{y}_{+}\right) \exp \left(i \hat{\mu}^{(0)} \hat{\xi}\right)
\end{aligned}
$$

The above relation yields an approximation to the growth rate $\hat{\mu}^{(0)}$ given a trial function for the transverse profile $A_{0}^{(0)}(\hat{x}, \hat{y})$. Since $\tilde{Q}_{0}^{(0)}\left(\hat{x}, \hat{y}, \hat{x}_{+}, \hat{y}_{+}, \hat{\xi}\right)=\tilde{Q}_{0}^{(0)}\left(\hat{x}_{+}, \hat{y}_{+}, \hat{x}, \hat{y}, \hat{\xi}\right)$, a first order variation of $A_{0}^{(0)}$ in the neighborhood of the actual solution yields an estimate for $\hat{\mu}^{(0)}$ that is accurate up to second order terms. For the fundamental mode, we use a Gaussian trial solution of the form $A_{0}^{(0)}(\hat{x}, \hat{y})=$ $\exp \left(-a_{x} \hat{x}^{2}-a_{y} \hat{y}^{2}\right)$. The resulting variational dispersion relation is

$$
\begin{aligned}
F_{0}\left(\hat{\mu}^{(0)}, a_{x}, a_{y}\right)= & \hat{\mu}^{(0)}+\eta_{d x} a_{x}+\eta_{d y} a_{y}+a_{x}^{1 / 2} a_{y}^{1 / 2} \\
& \times \int_{-\infty}^{0} d \hat{\xi} \hat{\xi} \exp \left[i\left(\hat{\mu}^{(0)}-\hat{\nu}\right) \hat{\xi}\right. \\
& \left.-\hat{\sigma}_{\delta}^{2} \hat{\xi}^{2} / 2\right] D_{0 x}^{-1 / 2} D_{0 y}^{-1 / 2}=0,
\end{aligned}
$$

where

$$
\begin{aligned}
& D_{0 x}=\frac{1}{4}\left(1+i \eta_{\varepsilon x} \hat{\xi}\right)^{2}+\left(1+i \eta_{\varepsilon x} \hat{\xi}\right) a_{x}+a_{x}^{2} \sin ^{2}\left(\hat{k}_{\beta x} \hat{\xi}\right), \\
& D_{0 y}=\frac{1}{4}\left(1+i \eta_{\varepsilon y} \hat{\xi}\right)^{2}+\left(1+i \eta_{\varepsilon y} \hat{\xi}\right) a_{y}+a_{y}^{2} \sin ^{2}\left(\hat{k}_{\beta y} \hat{\xi}\right) .
\end{aligned}
$$

The solution is completed by the relations $\partial F_{0} / \partial a_{x}=0$ and $\partial F_{0} / \partial a_{y}=0$, which are due to the fact that the stationary growth rate satisfies $\partial \hat{\mu}^{(0)} / \partial a_{x}=\partial \hat{\mu}^{(0)} /$ $\partial a_{y}=0$. These three equations yield the fundamental growth rate $\hat{\mu}^{(0)}$ and the mode parameters $a_{x}$ and $a_{y}$ as functions of the detuning $\hat{\nu}$.

For $p_{0} \neq 0$, the periodic $z$ dependence is restored and the two satellite amplitudes $A_{ \pm 1}$ need to be included in our analysis. Since $\tilde{d}_{ \pm 1}=O(\epsilon)$, we anticipate that the leading contribution to $A_{ \pm 1}$ will also be $\propto \epsilon$. The two first-order equations for $A_{ \pm 1}$ are

$$
\begin{aligned}
& \left(\hat{\mu}^{(0)} \pm \hat{k}_{\beta x}-\left[\eta_{d x} \frac{\partial^{2}}{\partial \hat{x}^{2}}+\eta_{d y} \frac{\partial^{2}}{\partial \hat{y}^{2}}\right]\right) A_{ \pm 1}^{(1)}(\hat{x}, \hat{y}) \\
& \quad-\int d \hat{x}_{+} d \hat{y}_{+} \int_{-\infty}^{0} d \hat{\xi} \tilde{Q}_{0}^{(0)}\left(\hat{x}, \hat{y}, \hat{x}_{+}, \hat{y}_{+}, \hat{\xi}\right) A_{ \pm 1}^{(1)}\left(\hat{x}_{+}, \hat{y}_{+}\right) \\
& \quad \times \exp \left[i\left(\hat{\mu}^{(0)} \pm \hat{k}_{\beta x}\right) \hat{\xi}\right]=F_{ \pm 1}(\hat{x}, \hat{y})=-\frac{i \epsilon_{1}}{2} \frac{\partial A_{0}^{(0)}(\hat{x}, \hat{y})}{\partial \hat{x}} \\
& \quad+\int d \hat{x}_{+} d \hat{y}_{+} \int_{-\infty}^{0} d \hat{\xi} \tilde{Q}_{ \pm 1}^{(1)}\left(\hat{x}, \hat{y}, \hat{x}_{+}, \hat{y}_{+}, \hat{\xi}\right) \\
& \quad \times A_{0}^{(0)}\left(\hat{x}_{+}, \hat{y}_{+}\right) \exp \left[i \hat{\mu}^{(0)} \hat{\xi}\right] .
\end{aligned}
$$

We note that-unlike a typical, homogeneous mode equation such as Eq. (58), which involves both an unknown growth rate and a transverse profile-the above results are driven equations for the transverse profile only. Given $\hat{\mu}^{(0)}$ and $A_{0}^{(0)}(\hat{x}, \hat{y})$ from the standard variational calculation described earlier, Eqs. (62) can also be solved using a modified version of the variational technique suitable for driven equations. In particular, we define the functional

$$
\begin{aligned}
I_{ \pm}= & \int d \hat{x} d \hat{y} A_{ \pm 1}^{(1)}(\hat{x}, \hat{y})\left\{\hat{\mu}^{(0)} \pm \hat{k}_{\beta x}-\left[\eta_{d x} \frac{\partial^{2}}{\partial \hat{x}^{2}}+\eta_{d y} \frac{\partial^{2}}{\partial \hat{y}^{2}}\right]\right\} \\
& \times A_{ \pm 1}^{(1)}(\hat{x}, \hat{y})-\int d \hat{x} d \hat{y} A_{ \pm 1}^{(1)}(\hat{x}, \hat{y}) \int d \hat{x}_{+} d \hat{y}_{+} \int_{-\infty}^{0} d \hat{\xi} \\
& \times \tilde{Q}_{0}^{(0)}\left(\hat{x}, \hat{y}, \hat{x}_{+}, \hat{y}_{+}, \hat{\xi}\right) A_{ \pm 1}^{(1)}\left(\hat{x}_{+}, \hat{y}_{+}\right) \exp \left[i\left(\hat{\mu}^{(0)} \pm \hat{k}_{\beta x}\right) \hat{\xi}\right] \\
& -\kappa \int d \hat{x} d \hat{y} A_{ \pm 1}^{(1)}(\hat{x}, \hat{y}) F_{ \pm 1}(\hat{x}, \hat{y}),
\end{aligned}
$$

where the driving term $F_{ \pm 1}(\hat{x}, \hat{y})$ has already been defined in Eq. (62) and $\kappa=2$. The above relation yields a complex number $I_{ \pm}$for a given profile $A_{ \pm 1}^{(1)}(\hat{x}, \hat{y})$. As is shown in the Appendix, in the neighborhood of the actual solution, $I_{ \pm}$is nonzero but stationary. In other words, a first order variation in $A_{ \pm 1}^{(1)}$ only yields a second order variation in $I_{ \pm}$. As is also argued in the Appendix, a suitable trial function for the satellite amplitudes is $A_{ \pm 1}^{(1)}(\hat{x}, \hat{y})=$ $\lambda_{ \pm} \hat{x} \exp \left(-b_{ \pm} \hat{x}^{2}\right) \exp \left(-c_{ \pm} \hat{y}^{2}\right)$. Substituting this form into Eq. (63) and also using the Gaussian trial function for $A_{0}^{(0)}(\hat{x}, \hat{y})$, we obtain the relation 


$$
\begin{aligned}
I_{ \pm}= & \frac{\pi \lambda_{ \pm}^{2}}{8 b_{ \pm} \sqrt{b_{ \pm}} \sqrt{c_{ \pm}}}\left[\hat{\mu}^{(0)} \pm \hat{k}_{\beta x}+3 \eta_{d x} b_{ \pm}+\eta_{d y} c_{ \pm}\right] \\
& +\frac{\pi \lambda_{ \pm}^{2}}{8} \int_{-\infty}^{0} d \hat{\xi} \hat{\xi} \exp \left[i\left(\hat{\mu}^{(0)} \pm \hat{k}_{\beta x}-\hat{\nu}\right) \hat{\xi}-\hat{\sigma}_{\delta}^{2} \hat{\xi}^{2} / 2\right] \\
& \times N_{1 x} D_{1 x}^{-3 / 2} D_{1 y}^{-1 / 2}-i \pi \epsilon_{1} \lambda_{ \pm} \frac{a_{x}}{\left(b_{ \pm}+a_{x}\right)^{3 / 2}} \frac{1}{\left(c_{ \pm}+a_{y}\right)^{1 / 2}} \\
& +\frac{i \pi \epsilon \lambda_{ \pm}}{4} \int_{-\infty}^{0} d \hat{\xi}^{2} \exp \left[i\left(\hat{\mu}^{(0)}-\hat{\nu}\right) \hat{\xi}-\hat{\sigma}_{\delta}^{2} \hat{\xi}^{2} / 2\right] \\
& \times N_{x} D_{x}^{-3 / 2} D_{y}^{-1 / 2},
\end{aligned}
$$

where

$$
\begin{aligned}
N_{x}= & a_{x} \exp \left( \pm i \hat{k}_{\beta x} \hat{\xi}\right) \sin \left(\hat{k}_{\beta x} \hat{\xi}\right) \pm \frac{i}{2}\left(1+i \eta_{\varepsilon x} \hat{\xi}\right), \\
D_{x}= & b_{ \pm} a_{x} \sin ^{2}\left(\hat{k}_{\beta x} \hat{\xi}\right)+\frac{1}{2}\left(1+i \eta_{\varepsilon x} \hat{\xi}\right)\left(b_{ \pm}+a_{x}\right) \\
& +\frac{\left(1+i \eta_{\varepsilon x} \hat{\xi}\right)^{2}}{4}, \\
D_{y}= & c_{ \pm} a_{y} \sin ^{2}\left(\hat{k}_{\beta y} \hat{\xi}\right)+\frac{1}{2}\left(1+i \eta_{\varepsilon y} \hat{\xi}\right)\left(c_{ \pm}+a_{y}\right) \\
& +\frac{\left(1+i \eta_{\varepsilon y} \hat{\xi}\right)^{2}}{4}
\end{aligned}
$$

and

$$
\begin{aligned}
& N_{1 x}=\left(1+i \eta_{\varepsilon x} \hat{\xi}\right) \cos \left(\hat{k}_{\beta x} \hat{\xi}\right), \\
& D_{1 x}=b_{ \pm}^{2} \sin ^{2}\left(\hat{k}_{\beta x} \hat{\xi}\right)+b_{ \pm}\left(1+i \eta_{\varepsilon x} \hat{\xi}\right)+\frac{1}{4}\left(1+i \eta_{\varepsilon x} \hat{\xi}\right)^{2} \\
& D_{1 y}=c_{ \pm}^{2} \sin ^{2}\left(\hat{k}_{\beta y} \hat{\xi}\right)+c_{ \pm}\left(1+i \eta_{\varepsilon y} \hat{\xi}\right)+\frac{1}{4}\left(1+i \eta_{\varepsilon y} \hat{\xi}\right)^{2}
\end{aligned}
$$

For this case, the variational relations are $\partial I_{ \pm} / \partial \lambda_{ \pm}=$ $\partial I_{ \pm} / \partial b_{ \pm}=\partial I_{ \pm} / \partial c_{ \pm}=0$. Solving these equations yields the satellite mode parameters $\left(\lambda_{ \pm}, b_{ \pm}\right.$, and $\left.c_{ \pm}\right)$. In view of the simple quadratic dependence of $I_{ \pm}$on $\lambda_{ \pm}$and $\epsilon, \epsilon_{1}$, it can be readily seen that the resulting value for $\lambda_{ \pm}$is a linear combination of $\epsilon$ and $\epsilon_{1}$. Thus, as expected, $\lambda_{ \pm}$and $A_{ \pm 1}^{(1)}$ are indeed first order quantities (recall that $\epsilon$ and $\epsilon_{1}$ are both proportional to $p_{0}$ ).

Having determined $\hat{\mu}^{(0)}, A_{0}^{(0)}$, and $A_{ \pm 1}^{(1)}$, we seek the second order corrections to $\hat{\mu}$. Indeed, it can be shown that there are no first order corrections to the above quantity, so we simply have $\hat{\mu}=\hat{\mu}^{(0)}+\hat{\mu}^{(2)}$. Reviewing the version of Eq. (52) for $n=0$, we collect all second order terms. The end result is

$$
\begin{aligned}
& \hat{\mu}^{(2)} A_{0}^{(0)}(\hat{x}, \hat{y})+\left(\hat{\mu}^{(0)}-\left[\eta_{d x} \frac{\partial^{2}}{\partial \hat{x}^{2}}+\eta_{d y} \frac{\partial^{2}}{\partial \hat{y}^{2}}\right]\right) A_{0}^{(2)}(\hat{x}, \hat{y}) \\
& \quad+\frac{i \epsilon_{1}}{2}\left[\frac{\partial A_{1}^{(1)}(\hat{x}, \hat{y})}{\partial \hat{x}}+\frac{\partial A_{-1}^{(1)}(\hat{x}, \hat{y})}{\partial \hat{x}}\right] \\
& =\int d \hat{x}_{+} d \hat{y}_{+} \int_{-\infty}^{0} d \hat{\xi} \tilde{Q}_{0}^{(0)}\left(\hat{x}, \hat{y}, \hat{x}_{+}, \hat{y}_{+}, \hat{\xi}\right)\left[A_{0}^{(2)}\left(\hat{x}_{+}, \hat{y}_{+}\right)\right. \\
& \left.\quad+i \hat{\mu}^{(2)} \hat{\xi} A_{0}^{(0)}\left(\hat{x}_{+}, \hat{y}_{+}\right)\right] \exp \left[i \hat{\mu}^{(0)} \hat{\xi}\right]+\mathcal{G}(\hat{x}, \hat{y}),
\end{aligned}
$$

where

$$
\begin{aligned}
\mathcal{G}(\hat{x}, \hat{y})= & \int d \hat{x}_{+} d \hat{y}_{+} \int_{-\infty}^{0} d \hat{\xi} \tilde{Q}_{0}^{(2)}\left(\hat{x}, \hat{y}, \hat{x}_{+}, \hat{y}_{+}, \hat{\xi}\right) \\
& \times A_{0}^{(0)}\left(\hat{x}_{+}, \hat{y}_{+}\right) \exp \left[i \hat{\mu}^{(0)} \hat{\xi}\right]+\int d \hat{x}_{+} d \hat{y}_{+} \int_{-\infty}^{0} d \hat{\xi} \\
& \times \tilde{Q}_{-1}^{(1)}\left(\hat{x}, \hat{y}, \hat{x}_{+}, \hat{y}_{+}, \hat{\xi}\right) A_{1}^{(1)}\left(\hat{x}_{+}, \hat{y}_{+}\right) \\
& \times \exp \left[i\left(\hat{\mu}^{(0)}+\hat{k}_{\beta x}\right) \hat{\xi}\right]+\int d \hat{x}_{+} d \hat{y}_{+} \\
& \times \int_{-\infty}^{0} d \hat{\xi} \tilde{Q}_{1}^{(1)}\left(\hat{x}, \hat{y}, \hat{x}_{+}, \hat{y}_{+}, \hat{\xi}\right) A_{-1}^{(1)}\left(\hat{x}_{+}, \hat{y}_{+}\right) \\
& \times \exp \left[i\left(\hat{\mu}^{(0)}-\hat{k}_{\beta x}\right) \hat{\xi}\right] .
\end{aligned}
$$

Here, $\quad \tilde{Q}_{ \pm 1}^{(1)}\left(\hat{x}, \hat{y}, \hat{x}_{+}, \hat{y}_{+}, \hat{\xi}\right)=\tilde{d}_{ \pm 1}{ }^{(1)} \times \tilde{\Pi}\left(\hat{x}, \hat{y}, \hat{x}_{+}, \hat{y}_{+}, \hat{\xi}\right)_{\hat{\nu}_{s} \rightarrow \hat{\nu}}$ while the second order term related to $\tilde{Q}_{0}$ is given by

$$
\begin{aligned}
& \tilde{Q}_{0}^{(2)}\left(\hat{x}, \hat{y}, \hat{x}_{+}, \hat{y}_{+}, \hat{\xi}\right) \\
& =\tilde{\Pi}\left(\hat{x}, \hat{y}, \hat{x}_{+}, \hat{y}_{+}, \hat{\xi}\right)_{\hat{\nu}_{s} \rightarrow \hat{\nu}} \\
& \quad \times\left[-\frac{i \xi}{4 \eta_{d x}} \epsilon_{1}^{2}-\frac{\epsilon^{2} \hat{\xi}^{2}}{4} \frac{\hat{x}_{+}^{2}+\hat{x}^{2}-2 \hat{x} \hat{x}_{+} \cos \left(\hat{k}_{\beta x} \hat{\xi}\right)}{\sin ^{2}\left(\hat{k}_{\beta x} \hat{\xi}\right)}\right] .
\end{aligned}
$$

In order to extract $\hat{\mu}^{(2)}$, we multiply Eq. (67) by $A_{0}^{(0)}(\hat{x}, \hat{y})$ and integrate over the scaled transverse position. After using Eq. (58), as well as some properties discussed in the Appendix, we obtain a formula for the second order correction to the growth rate:

$$
\begin{aligned}
\hat{\mu}^{(2)} & {\left[\int d \hat{x} d \hat{y}\left[A_{0}^{(0)}(\hat{x}, \hat{y})\right]^{2}-i \int d \hat{x} d \hat{y} A_{0}^{(0)}(\hat{x}, \hat{y}) \int d \hat{x}_{+} d \hat{y}_{+}\right.} \\
& \left.\times \int_{-\infty}^{0} d \hat{\xi} \hat{\xi} \tilde{Q}_{0}^{(0)}\left(\hat{x}, \hat{y}, \hat{x}_{+}, \hat{y}_{+}, \hat{\xi}\right) \exp \left[i \hat{\mu}^{(0)} \hat{\xi}\right] A_{0}^{(0)}\left(\hat{x}_{+}, \hat{y}_{+}\right)\right] \\
= & -\frac{i \epsilon_{1}}{2} \int d \hat{x} d \hat{y} A_{0}^{(0)}(\hat{x}, \hat{y})\left[\frac{\partial A_{1}^{(1)}(\hat{x}, \hat{y})}{\partial \hat{x}}+\frac{\partial A_{-1}^{(1)}(\hat{x}, \hat{y})}{\partial \hat{x}}\right] \\
& +\int d \hat{x} d \hat{y} A_{0}^{(0)}(\hat{x}, \hat{y}) \mathcal{G}(\hat{x}, \hat{y}),
\end{aligned}
$$

where $\mathcal{G}$ has been defined in Eq. (68). Substituting the analytical expressions for $A_{0}^{(0)}$ and $A_{ \pm 1}^{(1)}$ into Eq. (70), we obtain the following analytical result: 


$$
\begin{aligned}
{[1} & \left.+i a_{x}^{1 / 2} a_{y}^{1 / 2} \int_{-\infty}^{0} d \hat{\xi} \hat{\xi}^{2} e^{\Psi} D_{0 x}^{-1 / 2} D_{0 y}^{-1 / 2}\right] \hat{\mu}^{(2)} \\
= & -i \epsilon_{1} a_{x}^{3 / 2} a_{y}^{1 / 2}\left[\frac{\lambda_{+}}{\left(a_{x}+b_{+}\right)^{3 / 2}\left(a_{y}+c_{+}\right)^{1 / 2}}\right. \\
& \left.+\frac{\lambda_{-}}{\left(a_{x}+b_{-}\right)^{3 / 2}\left(a_{y}+c_{-}\right)^{1 / 2}}\right] \\
& +\frac{\epsilon^{2}}{4} a_{x}^{1 / 2} a_{y}^{1 / 2} \int_{-\infty}^{0} d \hat{\xi} \hat{\xi}^{3} e^{\Psi} N_{0 x} D_{0 x}^{-3 / 2} D_{0 y}^{-1 / 2} \\
& +\frac{i \epsilon_{1}^{2}}{4 \eta_{d x}} a_{x}^{1 / 2} a_{y}^{1 / 2} \int_{-\infty}^{0} d \hat{\xi} \hat{\xi}^{2} e^{\Psi} D_{0 x}^{-1 / 2} D_{0 y}^{-1 / 2} \\
& +\frac{i}{4} \epsilon \lambda_{+} \sqrt{a_{x}} \sqrt{a_{y}} \int_{-\infty}^{0} d \hat{\xi} \hat{\xi}^{2} e^{\Psi_{+}}\left(\tilde{N}_{x} D_{x}^{-3 / 2} D_{y}^{-1 / 2}\right)_{+} \\
& +\frac{i}{4} \epsilon \lambda_{-} \sqrt{a_{x}} \sqrt{a_{y}} \int_{-\infty}^{0} d \hat{\xi} \hat{\xi}^{2} e^{\Psi}\left(\tilde{N}_{x} D_{x}^{-3 / 2} D_{y}^{-1 / 2}\right)_{-},
\end{aligned}
$$

where $\Psi=i\left(\hat{\mu}^{(0)}-\hat{\nu}\right) \hat{\xi}-\hat{\sigma}_{\delta}^{2} \hat{\xi}^{2} / 2, \Psi_{ \pm}=\Psi \pm i \hat{k}_{\beta x} \hat{\xi}, N_{0 x}=$ $a_{x}+\left(1+i \eta_{\varepsilon x} \hat{\xi}\right) / 2$, and

$$
\tilde{N}_{x}=a_{x} \sin \left(\hat{k}_{\beta x} \hat{\xi}\right)+\frac{1}{2}\left[\sin \left(\hat{k}_{\beta x} \hat{\xi}\right) \mp i \cos \left(\hat{k}_{\beta x} \hat{\xi}\right)\right]\left(1+i \eta_{\varepsilon x} \hat{\xi}\right)
$$

Given $\hat{\mu}^{(0)}, a_{x}, a_{y}, \lambda_{ \pm}, b_{ \pm}$, and $c_{ \pm}$(all of which are available from our previous variational calculations), Eq. (71) yields the second order correction to $\hat{\mu}$. This completes our perturbation solution for the periodic problem.

\section{NUMERICAL EXAMPLES}

This section provides a brief numerical illustration of the theoretical methods outlined above. In particular, we compare the results of the linearized IVP solution with the output of the three-dimensional FEL simulation code GENESIS [19] for a set of standard, LCLS-like electron beam and undulator parameters. Next, we use the asymptotic portion of the linearized solution in order to validate the results of the periodic, eigenmodelike analysis. Lastly, the latter are also contrasted with the Tanaka gain length formula for a kicked beam. To begin with, we consider the set of FEL parameters given in Table I, which are similar to those of a typical hard $\mathrm{x}$-ray configuration for LCLS. In particular, they involve generating $8.2 \mathrm{keV}$ photons $(0.15 \mathrm{~nm}$ radiation wavelength) with a $14.3 \mathrm{GeV} e$ beam and a $3 \mathrm{~cm}$ period undulator (with $K_{0}=3.7$ ) while the typical average beta value of $30 \mathrm{~m}$ corresponds to a beam size of about $23 \mu \mathrm{m}$ (for a transverse normalized emittance of $0.5 \mathrm{~mm} \mathrm{mrad}$ ). Besides this baseline value, we will briefly consider two other average beta values, namely 15 and $60 \mathrm{~m}$, in order to explore the sensitivity of our results with respect to the size of the electron beam. For all three configurations, we assume a peak current of $3 \mathrm{kA}$ and an rms energy spread of $10^{-4}$.
TABLE I. Undulator and electron beam parameters.

\begin{tabular}{lc}
\hline \hline Parameter & Value \\
\hline Undulator parameter $K_{0}$ & 3.7 \\
Undulator period $\lambda_{u}$ & $3 \mathrm{~cm}$ \\
Beam energy $\gamma_{0} m_{e} c^{2}$ & $14.31 \mathrm{GeV}$ \\
Resonant wavelength $\lambda_{r}$ & $0.15 \mathrm{~nm}$ \\
Peak current $I_{p}$ & $3 \mathrm{kA}$ \\
Energy spread $\sigma_{\delta}$ & $10^{-4}$ \\
Normalized emittance $\gamma_{0} \epsilon_{x}=\gamma_{0} \epsilon_{y}$ & $0.5 \mu \mathrm{m}$ \\
Average beta value $\beta_{e x}=\beta_{e y}$ & $15 / 30 / 60 \mathrm{~m}$ \\
Electron beam size $\sigma_{x}=\sigma_{y}$ & $16.3 / 23.1 / 32.7 \mu \mathrm{m}$ \\
FEL parameter $\rho$ & $(6.8 / 5.4 / 4.3) \times 10^{-4}$ \\
\hline \hline
\end{tabular}

Beginning with the $30 \mathrm{~m}$ beta case, we assume a symmetric, on-axis, Gaussian input radiation with a Rayleigh length of $12 \mathrm{~m}$ and a waist situated at the undulator entrance $(z=0)$. As far as the various dimensionless parameters are concerned, we have $\hat{A}_{x}=3.4$, $\hat{B}_{x}=\hat{B}_{y}=0.747$, and $\hat{\sigma}_{\delta}=0.184$, while the initial mode parameters are $\hat{\beta}_{x}(0)=\hat{\beta}_{y}(0)=0.4$ and $\hat{\alpha}_{x}(0)=0$. Moreover, the specific frequency we are considering translates into a scaled detuning of $\hat{\nu}=-0.5$. Using Eqs. (23)(26), we obtain the linearized solution of the IVP for the setup described above and four kick angle values corresponding to $\hat{p}_{0}=p_{0} / \sigma_{x}^{\prime}=0,1,2,3$ and $\hat{z}_{e}=0$ $\left(\sigma_{x}^{\prime}=\sigma_{x} / \beta_{e x}=0.77 \mu \mathrm{rad}\right)$. The principal results are presented in Fig. 1, in which we plot the $e$-beam horizontal centroid $x_{c e}$, FEL gain $G=\log \left(P_{r} / P_{0}\right)\left(P_{0}=4 \mathrm{~kW}\right.$ is the input power), and bunching factor $\left|\left\langle e^{-i \theta}\right\rangle\right|$ as functions of $z$. Also included for comparison are the corresponding results from a time-independent (steady-state) GENESIS run. Up to the onset of saturation, we observe good agreement between the linearized solution and the simulation results. In fact, the extent of the linear regime can be roughly estimated by simply determining the $z$ value for which the analytical result for the bunching factor becomes equal to unity.

A similar compatibility between the IVP solution and simulation can be established for the radiation beam size, as is shown in Fig. 2 (this particular example corresponds to a kick angle of $1.54 \mu \mathrm{rad}$ ). We clarify that the radiation size plotted here as a function of $z$ is a combined quantity defined by $\sigma_{r}=\sqrt{\left\langle x^{2}\right\rangle-\langle x\rangle^{2}+\left\langle y^{2}\right\rangle}$ (its analog in the context of the single-mode, Gaussian approximation is $\sigma_{r}=\sqrt{\sigma_{r x}^{2}+\sigma_{r y}^{2}}$ ). Moreover, Fig. 3 compares the horizontal centroid of the radiation beam as calculated from the linearized solution with the centroid of the $e$-beam. We observe that, after an initial delay due to the angular mismatch, the radiation centroid generally follows the oscillating electron beam.

Having established the basic validity of the linearized solution, we now turn to the task of integrating it with the formalism of the periodic analysis. Specifically, the 


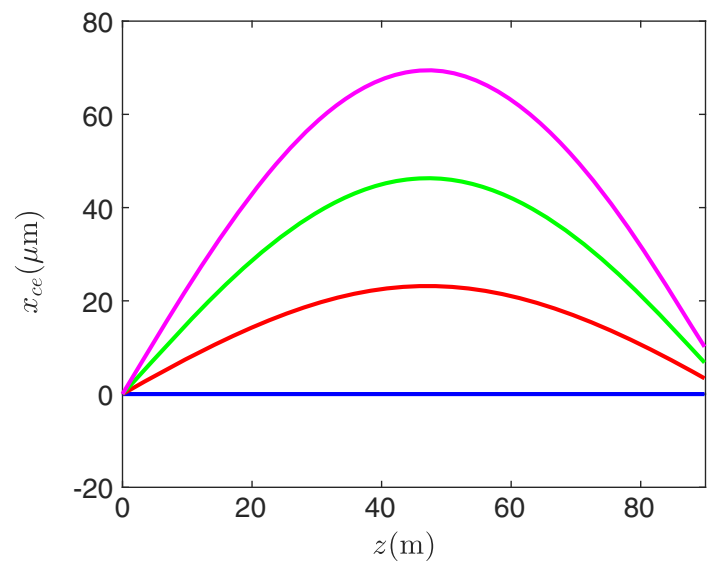

(a) Horizontal centroid $x_{c e}$ of the kicked e-beam

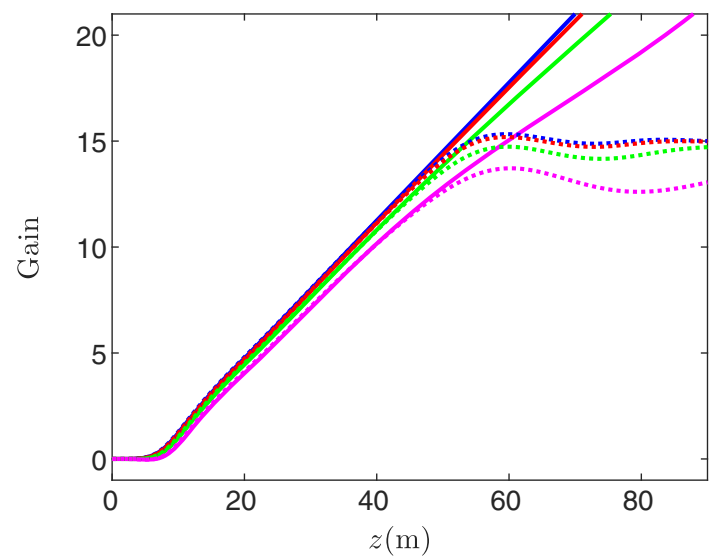

(b) FEL gain $G=\log \left(P_{r} / P_{0}\right)$.

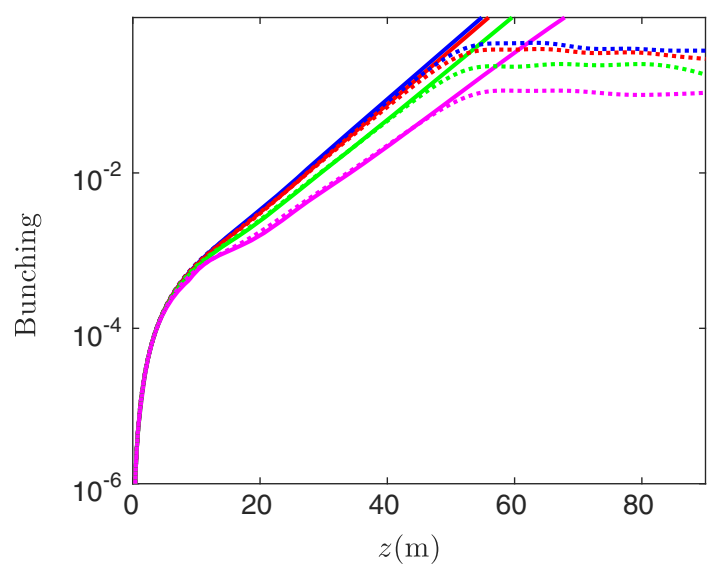

(c) Bunching factor $\left|\left\langle e^{-i \theta}\right\rangle\right|$.

FIG. 1. Electron beam centroid, FEL gain and bunching factor as functions of $z$, for a kick angle of $p_{0}=0 / 0.77 / 1.54 /$ $2.31 \mu \mathrm{rad}$ (blue/red/green/magenta). The solid and dashed lines represent the linearized solution and GENESIS simulation results, respectively.

principal task is to use Eqs. (60), (64), and (71) in order to determine the total average growth rate $\hat{\mu}=\hat{\mu}^{(0)}+\hat{\mu}^{(2)}$ in terms of the frequency detuning variable $\hat{\nu}$. The relevant dimensionless parameters (again assuming a $30 \mathrm{~m}$ beta

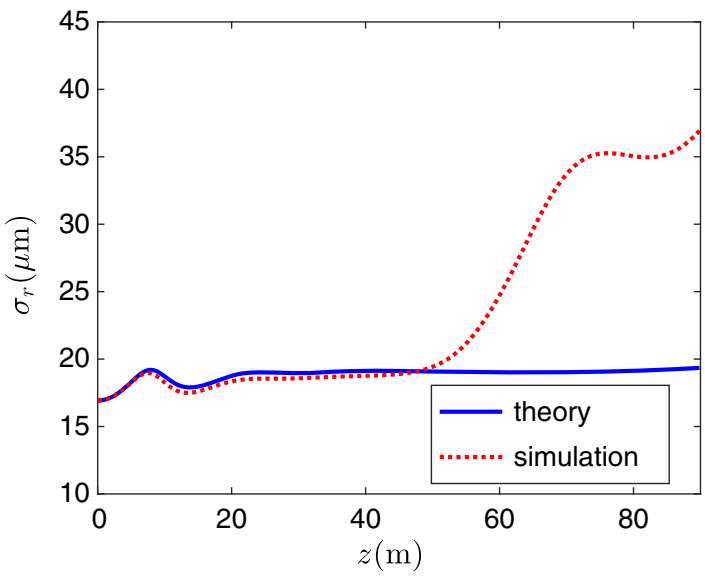

FIG. 2. Radiation beam size versus $z$ for a kick angle of $1.54 \mu \mathrm{rad}$ (theory and GENESIS simulation results).

value) are $\eta_{d x}=\eta_{d y}=0.098, \eta_{\varepsilon x}=\eta_{\varepsilon y}=0.109$, and $\hat{k}_{\beta x}=$ $\hat{k}_{\beta y}=0.146$ while $\epsilon=0.109 / 0.2$ and $\epsilon_{1}=0.145 / 0.29$ for a $0.77 / 1.54 \mu \mathrm{rad}$ kick. On the other hand, according to the discussion at the end of the orthogonal expansion subsection, the results of the single-mode IVP solution can be used in calculating a $z$-dependent, local, complex growth rate $\mu_{z}$ [see Eq. (39)] the negative imaginary part of which is equal to half the logarithmic power growth rate $P_{r}^{-1} d P_{r} / d z$. Thus, a direct comparison can be made between $-\hat{\mu}_{i}$ and $P_{r}^{-1} d P_{r} / d z=-2 \mu_{z i}$ scaled by $4 \rho k_{u}$. This is presented in Fig. 4 for the above-mentioned kick angle values. Each comparison includes both the unperturbed mode growth rate $\hat{\mu}^{(0)}$ and the "corrected" version which involves the second order contribution due to the kick (the latter is derived from the full, three-amplitude calculation described in the theory section). For both cases, it is evident that the scaled logarithmic power growth rate exhibits a periodic variation with $z$ in the asymptotic portion of the linear regime. Its averaged value (after excluding the initial transient) agrees rather well with

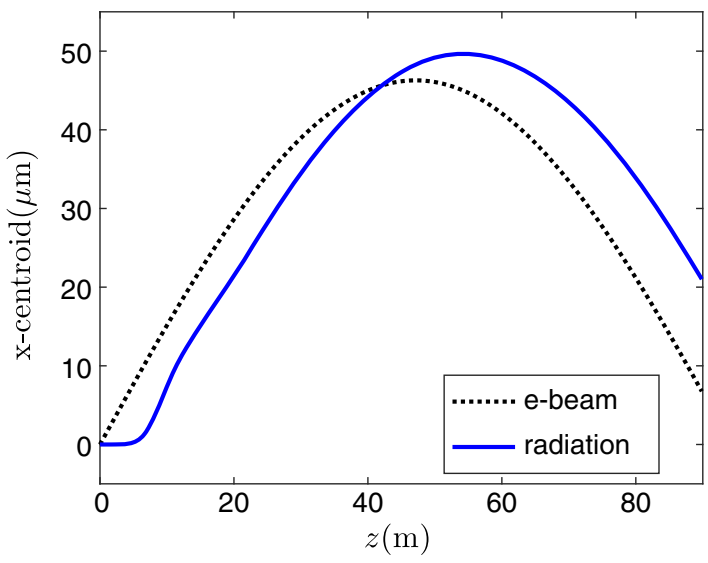

FIG. 3. Electron and radiation horizontal centroids for a kick angle of $1.54 \mu \mathrm{rad}$ (analytical results). 


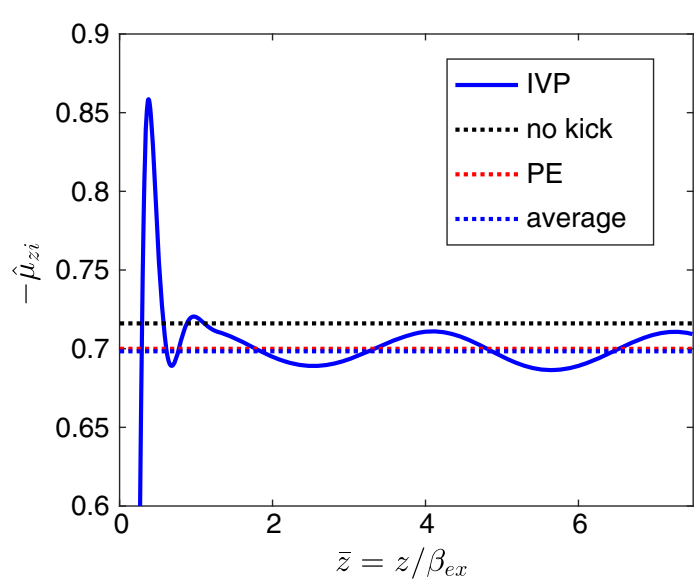

(a) $0.77 \mu \mathrm{rad}$ kick.

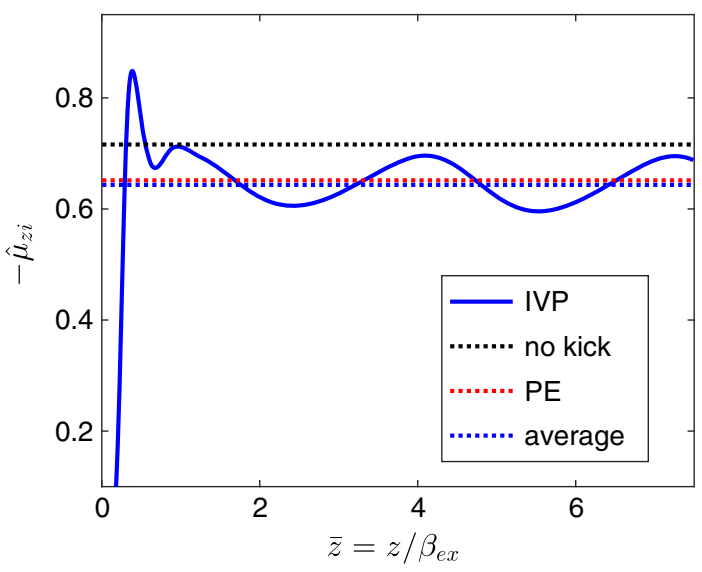

(b) $1.54 \mu \mathrm{rad}$ kick.

FIG. 4. Comparison between the scaled, local power growth rate $-\hat{\mu}_{z i}=-\mu_{z i} /\left(2 \rho k_{u}\right)$ and the results of the periodic analysis (PE). Also shown is the growth rate in the absence of a kick (results for $\beta_{e x}=30 \mathrm{~m}$ and $\hat{\nu}=-0.5$, as in Figs. 1-3).

the value calculated from the periodic analysis. Thus, we are encouraged to use the latter as a figure of merit for a kicked beam configuration (though saturation may sometimes limit the actual number of completed betatron oscillations).

To facilitate a proper comparison between the results of the periodic analysis and the Tanaka gain length formula, we first define the frequency-dependent coefficient $c_{\nu}=-\left(\hat{\mu}_{i}^{(2)} / \hat{\mu}_{i}^{(0)}\right) / \epsilon_{1}^{2}$. Since $\epsilon_{1} \propto p_{0}$ and $\hat{\mu}^{(2)} \propto p_{0}^{2}$, this quantity is independent of the kick angle. In fact, it is proportional to the coefficient of the quadratic decrease of the growth rate with respect to the angular kick. Figure 5(a) shows the plot of $c_{\nu}$ versus the scaled detuning $\hat{\nu}$, also comparing it with the corresponding value derived from Tanaka's formula [Eq. (1)]. The latter can be determined as follows: we recall that the power gain length $L_{G}$ is defined as the length necessary for one exponentiation of the radiation power. Since the power is proportional to $\left|E_{\nu}\right|^{2}$, for a field dependence of the form $E_{\nu} \propto \exp (i \mu z)$ (where $\mu$

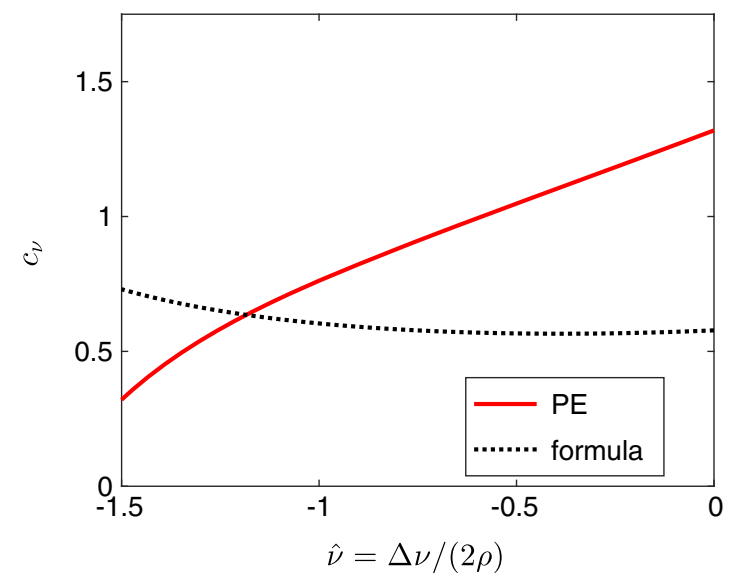

(a) Frequency-dependent coefficient $c_{\nu}=-\left(\hat{\mu}_{i}^{(2)} / \hat{\mu}_{i}^{(0)}\right) / \epsilon_{1}^{2}$.

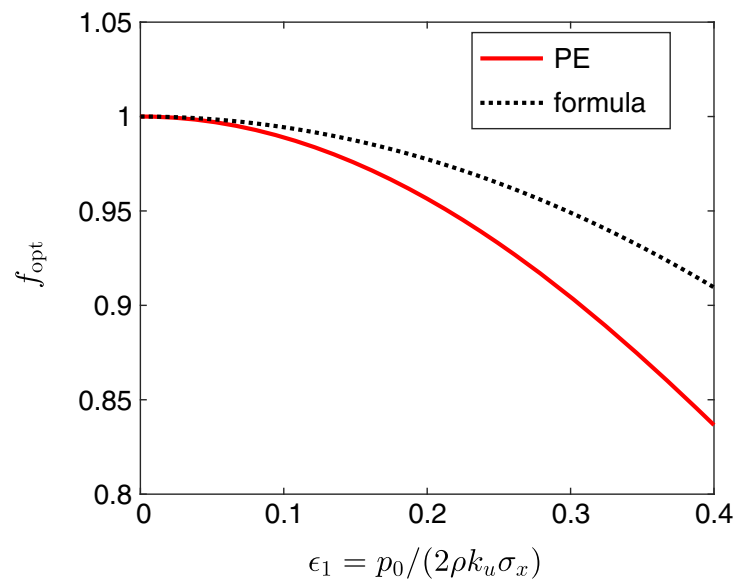

(b) Optimized coefficient $f_{\mathrm{opt}}=\left(\hat{\mu}_{i}^{(0)}+\hat{\mu}_{i}^{(2)}\right)_{\mathrm{opt}} /\left(\hat{\mu}_{i}^{(0)}\right)_{\mathrm{opt}}$.

FIG. 5. Comparison of the periodic analysis (PE) results with the estimates derived from the Tanaka formula [Eq. (1)] for $\beta_{e x}=30 \mathrm{~m}$. A stronger-than-anticipated reduction of the growth rate is observed.

is the complex growth rate), the gain length is given by $L_{G}=-1 /\left(2 \mu_{i}\right)$. Thus, in terms of our scaling, the gain length (in the absence of a kick) $L_{G 0}$ can be written as $L_{G 0}=f_{3 D} L_{0}$, where $L_{0}=\lambda_{u} /(4 \pi \sqrt{3} \rho)$ is the $1 \mathrm{D}$ gain length and $f_{3 D}=-\sqrt{3} /\left(2 \hat{\mu}_{i}^{(0)}\right)>1$ is a parameter expressing the influence of energy spread and 3D effects. Using Eq. (1), it is straightforward to show that the fractional growth rate decrease due to the kick is equal to $\left(p_{0} / \theta_{c}\right)^{2}=T_{F} \epsilon_{1}^{2}$, where $T_{F}=f_{3 D} /\left(4 \pi \sqrt{3} \eta_{d x}\right)$ is the quantity we plot in Fig. 5(a) (recall that the critical angle is given by $\left.\theta_{c}=\sqrt{\lambda_{r} / L_{G 0}}\right)$. Overall, we observe a somewhat stronger reduction in gain compared to what we would expect from the Tanaka formula.

A similar comparison involves the optimized coefficient $f_{\text {opt }} \equiv\left(\hat{\mu}_{i}^{(0)}+\hat{\mu}_{i}^{(2)}\right)_{\text {opt }} /\left(\hat{\mu}_{i}^{(0)}\right)_{\text {opt }}$, where both numerator and denominator have been maximized with respect to the detuning (a scenario relevant for self-amplified spontaneous 
emission). This quantity represents the fractional reduction in the optimized growth rate due to a kick of given strength. In Fig. 5(b), we plot $f_{\text {opt }}$ versus the $\epsilon_{1}$ parameter and compare its variation with that given by the gain length formula. The latter is simply $1-\left(p_{0} / \theta_{c}\right)^{2}=1-f_{3 D} \epsilon_{1}^{2} /\left(4 \pi \sqrt{3} \eta_{d x}\right)$, with $f_{3 D}$ involving the frequency-optimized value of the unperturbed growth rate $\hat{\mu}^{(0)}$. Once again, a stronger gain reduction (compared to the formula value) is observed. This conclusion can be quantified in a clearer way by recalling that $f_{\text {opt }}$ is equal to the ratio $L_{G 0} / L_{G}$. Thus, we can rewrite our results as

$$
L_{G}=\frac{L_{G 0}}{1-\alpha_{m}\left(p_{0} / \theta_{c}\right)^{2}},
$$

where the dimensionless coefficient $\alpha_{m}$ expresses the deviation from the value calculated by means of Eq. (1). In fact, we have $\alpha_{m} \approx \mathbf{2}$, for the $\mathbf{3 0} \mathbf{m}$ beta value, so that the fractional increase in the gain length is twice as much as that given by the Tanaka formula. An entirely similar study can be performed for the average beta values of $15 \mathbf{~ m}$ and $60 \mathrm{~m}$. The basic compatibility between the linearized IVP solution, 3D simulation and periodic analysis is still preserved while $\alpha_{m}$ is approximately equal to $\mathbf{2 . 5}$ and $\mathbf{1 . 5}$, respectively.

\section{CONCLUSIONS}

In this paper, we have developed a self-consistent theory for treating the influence of an angular kick on the operation of a high-gain FEL in the linear regime of the interaction. Working within the framework of a Maxwell-Vlasov formalism, we derived a single, three-dimensional equation that governs the evolution of the slowly varying radiation amplitude along the undulator, taking into account the effect of the oscillating $e$-beam centroid. Using an expansion of the radiation amplitude in terms of an appropriate set of orthogonal transverse modes, we obtained a semianalytical, approximate solution to the linearized initial value problem which includes the calculation of key $e$-beam and radiation parameters. The validity of these linearized results is established through direct comparison with simulation for an LCLS-like FEL configuration. Furthermore, we presented a periodic analysis technique which allows us to define and determine an averaged FEL growth rate, suitable for describing the kickedbeam setup after averaging over the centroid oscillations. After verifying its results through a comparison with the asymptotic part of the linearized IVP solution, the periodic analysis is in turn contrasted with Tanaka's gain length formula, revealing a stronger than expected quadratic decrease of the growth rate with the kick angle.

\section{ACKNOWLEDGMENTS}

This work was supported by the Director, Office of Science, of the U.S. Department of Energy under Contract No. DE-AC02-76SF00515.

\section{APPENDIX: VARIATIONAL TECHNIQUE FOR DRIVEN EQUATIONS}

A typical FEL eigenmode problem is defined by a homogeneous equation of the form

$$
\left(\mu_{0}+\lambda_{0} \nabla_{\perp}^{2}\right) \hat{A}_{0}(\mathbf{x})-\int d^{2} \mathbf{x}_{+} G_{K}\left(\mathbf{x}, \mathbf{x}_{+}, \mu_{0}\right) \hat{A}_{0}\left(\mathbf{x}_{+}\right)=0
$$

where $\hat{A}_{0}(\mathbf{x})$ is the unknown FEL mode profile, $\mu_{0}$ is the corresponding complex growth rate (also to be determined), and $\lambda_{0}$ is a constant. The standard variational technique for such a problem is based on constructing the functional relation expressed by

$$
\begin{aligned}
& \int d^{2} \mathbf{x} \hat{A}(\mathbf{x})\left[\left(\mu+\lambda_{0} \nabla_{\perp}^{2}\right) \hat{A}(\mathbf{x})\right. \\
& \left.-\int d^{2} \mathbf{x}_{+} \hat{A}\left(\mathbf{x}_{+}\right) G_{K}\left(\mathbf{x}, \mathbf{x}_{+}, \mu\right)\right]=0,
\end{aligned}
$$

which yields a complex number $\mu$ given a function $\hat{A}(\mathbf{x})$. It is evident that substituting the exact mode profile [that is, $\left.\hat{A}(\mathbf{x})=\hat{A}_{0}(\mathbf{x})\right]$ into Eq. (A2) yields the exact growth rate (i.e. $\mu=\mu_{0}$ ). It can also be shown that, provided that $G_{K}\left(\mathbf{x}, \mathbf{x}_{+}, \mu_{0}\right)=G_{K}\left(\mathbf{x}_{+}, \mathbf{x}, \mu_{0}\right)$, a first order variation around $A_{0}(\mathbf{x})$ actually results in a second order variation around $\mu_{0}$ [13]. The details of this proof are similar to the development presented below so they will be omitted. The variational solution is thus based on the functional of Eq. (A2) and a proper choice of trial function.

The question we aim to address is whether a variational solution can also be found for a driven equation of the form

$$
\begin{aligned}
& \left(\mu_{0}+\lambda_{0} \nabla_{\perp}^{2}\right) \hat{A}_{0}(\mathbf{x}) \\
& \quad-\int d^{2} \mathbf{x}_{+} G_{K}\left(\mathbf{x}, \mathbf{x}_{+}, \mu_{0}\right) \hat{A}_{0}\left(\mathbf{x}_{+}\right)=F(\mathbf{x}),
\end{aligned}
$$

where $\hat{A}_{0}(\mathbf{x})$ is the unknown mode profile and $\mu_{0}$ is now a known complex constant (both unrelated to the corresponding parameters of the homogeneous problem). We begin by constructing the functional

$$
\begin{aligned}
I= & \int d^{2} \mathbf{x} \hat{A}(\mathbf{x})\left[\left(\mu_{0}+\lambda_{0} \nabla_{\perp}^{2}\right) \hat{A}(\mathbf{x})\right. \\
& \left.-\int d^{2} \mathbf{x}_{+} \hat{A}\left(\mathbf{x}_{+}\right) G_{K}\left(\mathbf{x}, \mathbf{x}_{+}, \mu_{0}\right)-\kappa F(\mathbf{x})\right],
\end{aligned}
$$

where $\kappa$ is another constant. Given a trial function $\hat{A}(\mathbf{x})$, one obtains a complex number $I$. In view of Eq. (A3), for $\hat{A}(\mathbf{x})=\hat{A}_{0}(\mathbf{x})$, we have

$$
I=I_{0}=(1-\kappa) \int d^{2} \mathbf{x} \hat{A}_{0}(\mathbf{x}) F(\mathbf{x}),
$$


which is generally nonzero for $\kappa \neq 1$. Now, we consider the first order variation of $I$. For $\hat{A}(\mathbf{x}) \rightarrow \hat{A}_{0}(\mathbf{x})+\hat{A}_{1}(\mathbf{x})$, we have $I \rightarrow I_{0}+I_{1}$, with

$$
\begin{aligned}
I_{1}= & \int d^{2} \mathbf{x}\left\{2 \mu_{0} \hat{A}_{0}(\mathbf{x}) \hat{A}_{1}(\mathbf{x})+\lambda_{0} \hat{A}_{0}(\mathbf{x}) \nabla_{\perp}^{2} \hat{A}_{1}(\mathbf{x})\right. \\
& \left.+\lambda_{0} \hat{A}_{1}(\mathbf{x}) \nabla_{\perp}^{2} \hat{A}_{0}(\mathbf{x})\right\}-\int d^{2} \mathbf{x} \int d^{2} \mathbf{x}_{+} G_{K}\left(\mathbf{x}, \mathbf{x}_{+}, \mu_{0}\right) \\
& \times\left\{\hat{A}_{0}(\mathbf{x}) \hat{A}_{1}\left(\mathbf{x}_{+}\right)+\hat{A}_{1}(\mathbf{x}) \hat{A}_{0}\left(\mathbf{x}_{+}\right)\right\} \\
& -\int d^{2} \mathbf{x} \kappa F(\mathbf{x}) \hat{A}_{1}(\mathbf{x}) .
\end{aligned}
$$

Next, we use the properties

$$
\int d^{2} \mathbf{x} \hat{A}_{0}(\mathbf{x}) \nabla_{\perp}^{2} \hat{A}_{1}(\mathbf{x})=\int d^{2} \mathbf{x} \hat{A}_{1}(\mathbf{x}) \nabla_{\perp}^{2} \hat{A}_{0}(\mathbf{x})
$$

which come from integration by parts, and

$$
\begin{aligned}
& \int d^{2} \mathbf{x} \int d^{2} \mathbf{x}_{+} \hat{A}_{0}(\mathbf{x}) \hat{A}_{1}\left(\mathbf{x}_{+}\right) G_{K}\left(\mathbf{x}, \mathbf{x}_{+}, \mu_{0}\right) \\
& =\int d^{2} \mathbf{x}_{+} \int d^{2} \mathbf{x} \hat{A}_{0}\left(\mathbf{x}_{+}\right) \hat{A}_{1}(\mathbf{x}) G_{K}\left(\mathbf{x}_{+}, \mathbf{x}, \mu_{0}\right) \\
& =\int d^{2} \mathbf{x} \int d^{2} \mathbf{x}_{+} \hat{A}_{1}(\mathbf{x}) \hat{A}_{0}\left(\mathbf{x}_{+}\right) G_{K}\left(\mathbf{x}, \mathbf{x}_{+}, \mu_{0}\right)
\end{aligned}
$$

which is based on the fact that the integral kernel satisfies $G_{K}\left(\mathbf{x}_{+}, \mathbf{x}, \mu_{0}\right)=G_{K}\left(\mathbf{x}, \mathbf{x}_{+}, \mu_{0}\right)$. With these in mind, we find

$$
\begin{aligned}
I_{1}= & \int d^{2} \mathbf{x} 2 \hat{A}_{1}(\mathbf{x})\left\{\mu_{0} \hat{A}_{0}(\mathbf{x})+\lambda_{0} \nabla_{\perp}^{2} \hat{A}_{0}(\mathbf{x})\right. \\
& \left.-\int d^{2} \mathbf{x}_{+} \hat{A}_{0}\left(\mathbf{x}_{+}\right) G_{K}\left(\mathbf{x}, \mathbf{x}_{+}, \mu_{0}\right)-\frac{\kappa}{2} F(\mathbf{x})\right\} \\
= & (2-\kappa) \int d^{2} \mathbf{x} \hat{A}_{1}(\mathbf{x}) F(\mathbf{x}) .
\end{aligned}
$$

If we select $\kappa=2$, we have $I_{0} \neq 0$ but $I_{1}=0$ i.e. the functional gives a nonzero but stationary value. Thus, a variational solution can be constructed even for nonhomogeneous equations. All of the above conclusions still hold when $\nabla_{\perp}^{2}$ is replaced by any linear operator $\mathcal{L}$ for which $\int d^{2} \mathbf{x} \hat{A}_{0}(\mathbf{x}) \mathcal{L} \hat{A}_{1}(\mathbf{x})=\int d^{2} \mathbf{x} \hat{A}_{1}(\mathbf{x}) \mathcal{L} \hat{A}_{0}(\mathbf{x})$. In our case, the relevant operator is $\mathcal{L}=\eta_{d x} \partial^{2} / \partial x^{2}+\eta_{d y} \partial^{2} / \partial y^{2}$. This particular form is involved in all the mode equations encountered in the periodic analysis of the main text. This includes both the homogeneous result-namely, Eq. (58)—and the driven versions [Eqs. (62)].

As far as the specific form of the variational trial functions is concerned, we have the following arguments: for nondriven mode equations such as Eq. (A1), we deem it sufficient to use a standard trial function of the form
$\hat{A}_{0}(x, y)=\exp \left(-a_{x} x^{2}\right) \exp \left(-a_{y} y^{2}\right)$ for the fundamental FEL mode, whenever the integral kernel is characterized by a Gaussian transverse dependence [see, for example, the relevant expression in Eq. (58)]. This choice is standard practice for an electron beam with a Gaussian transverse profile and can be justified on the basis of some of the arguments given below. For the driven mode equations such as Eq. (A3), we instead elect to use a modified trial function of the form $\hat{A}_{0}(x, y)=\lambda x \exp \left(-b x^{2}\right) \exp \left(-c y^{2}\right)$.

The reasoning for the latter choice can be stated as follows: suppose $\hat{A}_{0}$ and $\mu_{0}$ satisfy a homogeneous mode equation of the form

$$
\left(\mu_{0}+\lambda_{0} \nabla_{\perp}^{2}\right) \hat{A}_{0}(\mathbf{x})-U\left(\mathbf{x}, \mu_{0}\right) \hat{A}_{0}(\mathbf{x})=0 .
$$

In fact, this is the limiting form of the general mode equation for the parallel beam regime (negligible emittance and focusing effects). If $U$ has a parabolic dependence of the form $U(x, y)=u_{0}-u_{1} x^{2}-u_{2} y^{2}$, the exact solution for the fundamental mode is actually an asymmetric Gaussian like the one mentioned above $[13,20]$. This result is valid only when the diffraction parameters $\eta_{d x}$ and $\eta_{d y}$ are smaller than unity, in which case the radiation sizes are smaller than the corresponding $e$-beam sizes and the cutoff of the parabolic profile can be ignored. Now consider the function $\Phi=\lambda x \hat{A}_{0}$, where $\lambda$ is a constant. It is easily shown that $\Phi$ satisfies the driven equation

$$
\left(\mu_{0}+\lambda_{0} \nabla_{\perp}^{2}\right) \Phi-U\left(\mathbf{x}, \mu_{0}\right) \Phi=2 \lambda_{0} \lambda \partial \hat{A}_{0} / \partial x .
$$

An inspection of Eqs. (62) shows that the above form is entirely analogous to the parallel beam limit of the driven equations given in the main text. Indeed, for $\sigma_{x}^{\prime} \rightarrow 0$ and $\sigma_{y}^{\prime} \rightarrow 0$, we have $\hat{k}_{\beta x}, \hat{k}_{\beta y} \rightarrow 0, \eta_{\varepsilon x}, \eta_{\varepsilon y} \rightarrow 0, \epsilon \rightarrow 0$, and $\tilde{Q}_{ \pm 1}^{(1)} \rightarrow 0$, so the driving term on the right-hand side of Eqs. (62) is simply proportional to $\partial A_{0}^{(0)} / \partial \hat{x}$ while the differential operators on the left-hand side of Eqs. (58) and (62) are identical. In other words, the only remaining driving term is proportional to the $x$ derivative of the solution to the homogeneous equation, which is precisely the case we described above. To recapitulate, in the parallel beam regime, the solution to the driven problem is simply the homogeneous solution (an asymmetric Gaussian) multiplied by $x$ and a constant. We use the same functional form to model our trial function for the driven equation in the general case.

[1] W. Ackermann et al., Operation of a free-electron laser from the extreme ultraviolet to the water window, Nat. Photonics 1, 336 (2007).

[2] P. Emma et al., First lasing and operation of an ångstromwavelength free-electron laser, Nat. Photonics 4, 641 (2010). 
[3] T. Ishikawa et al., A compact X-ray free-electron laser emitting in the sub-ångström region, Nat. Photonics 6, 540 (2012).

[4] E. Allaria et al., Highly coherent and stable pulses from the FERMI seeded free-electron laser in the extreme ultraviolet, Nat. Photonics 6, 699 (2012).

[5] J. Krzywinski (personal communication).

[6] P. Emma, SLAC theory (unpublished).

[7] A. Lutman et al., Polarization control in an X-ray freeelectron laser, Nat. Photonics 10, 468 (2016).

[8] H. D. Nuhn (personal communication).

[9] T. Tanaka et.al, Consideration on the BPM alignment tolerance in X-ray FELs, Nucl. Instrum. Methods Phys. Res., Sect. A 528, 172 (2004).

[10] Z. Huang and K.-J. Kim, Review of x-ray free-electron laser theory, Phys. Rev. ST Accel. Beams 10, 034801 (2007).

[11] E. L. Saldin, E. A. Schneidmiller, and M. V. Yurkov, The Physics of Free Electron Lasers (Springer-Verlag, Berlin, 2000).

[12] G. Geloni, V. Kocharyan, and E. Saldin, Effect of Aberration of Light in X-ray Free Electron Lasers, arXiv:1511.01375.

[13] P. Baxevanis, Ph.D. thesis, Stanford University, 2015.
[14] R. Bonifacio, C. Pellegrini, and L. Narducci, Collective instabilities and high-gain regime in a free electron laser, Opt. Commun. 50, 373 (1984).

[15] P. Baxevanis and R. Ruth, Initial value problem for an FEL driven by an asymmetric electron beam, 36th International Free Electron Laser Conference, Basel, Switzerland, 2014 (2014), p. 433, http://accelconf.web.cern.ch/AccelConf/ FEL2014/papers/tup027.pdf.

[16] P. Sprangle, A. Ting, and C. M. Tang, Analysis of radiation focusing and steering in the free-electron laser by use of a source-dependent expansion technique, Phys. Rev. A 36, 2773 (1987).

[17] P. Baxevanis, R. Ruth, and Z. Huang, General method for analyzing three-dimensional effects in free-electron laser amplifiers, Phys. Rev. ST Accel. Beams 16, 010705 (2013).

[18] M. Xie, High gain free electron lasers driven by flat electron beam, Nucl. Instrum. Methods Phys. Res., Sect. A 507, 450 (2003).

[19] S. Reiche, GENESIS 1.3: A fully 3D time-dependent FEL simulation code, Nucl. Instrum. Methods Phys. Res., Sect. A 429, 243 (1999).

[20] M Xie, Ph.D. thesis, Stanford University, 1988. 